\title{
Retarded field calculation of electron energy loss in inhomogeneous dielectrics
}

\author{
F. J. García de Abajo* \\ Centro Mixto CSIC-UPV/EHU and Donostia International Physics Center (DIPC), Aptdo. 1072, 20080 San Sebastián, Spain
}

\section{A. Howie}

Cavendish Laboratory, Madingley Road, Cambridge CB3 OHE, England

(Received 21 September 2001; published 8 March 2002)

\begin{abstract}
The exact solution of Maxwell's equations in the presence of arbitrarily shaped dielectrics is expressed in terms of surface-integral equations evaluated at the interfaces. The electromagnetic field induced by the passage of an external electron is then calculated in terms of self-consistently obtained boundary charges and currents. This procedure is shown to be suitable for the simulation of electron energy loss spectra when the materials under consideration are described by local frequency-dependent response functions. The particular cases of translationally invariant interfaces and axially symmetric interfaces are discussed in detail. The versatility of this method is emphasized by examples of energy loss spectra for electrons passing near metallic and dielectric wedges, coupled cylinders, spheres, and tori, and other complex geometries, where retardation aspects and Cherenkov losses can sometimes be significant.
\end{abstract}

DOI: 10.1103/PhysRevB.65.115418 PACS number(s): 73.20.Mf, 71.45.Gm, 41.20.-q, 79.20.-m

\section{INTRODUCTION}

Spatially resolved energy loss spectroscopy with fast electrons has proved to be a powerful microscopy technique for determining local chemical and electronic structure, particularly when looking at the excitation of target atomic cores. ${ }^{1}$ The relatively more intense low-energy, valence excitation part of the loss spectrum is also of considerable interest, ${ }^{2-5}$ but so far has been of less practical importance. The larger impact parameters associated with low-energy transfers can result in reduced spatial resolution and the theory needed to interpret the results is more complex and less direct.

In this context, the surface modes of various particularly simple geometries have been theoretically investigated in the past without inclusion of retardation effects (e.g., in planar surfaces $^{6}$ and in sharp, ${ }^{7,8}$ hyperbolic, ${ }^{8}$ and parabolic ${ }^{9}$ wedges). Fast-electron energy losses have been analytically calculated, also in the nonretarded limit, near planar surfaces, ${ }^{10,11}$ isolated spheres, ${ }^{12-14}$ hyperbolic wedges, ${ }^{15}$ spheroids, ${ }^{16}$ cylinders,${ }^{17}$ two coupled cylinders, ${ }^{18}$ combinations of spheres and planes, ${ }^{19-21}$ and more recently, near two coupled spheres. ${ }^{22}$ Granular materials composed of randomly distributed spheres have been studied as well using effective medium theories to find the loss function of equivalent homogeneous media. ${ }^{23-26}$

For more complex geometries often encountered in practice, numerical simulations of the excitations generated by a passing electron are necessary and have already been successfully explored in the nonretarded case. ${ }^{27-32}$ This type of calculation was first introduced by Fuchs ${ }^{27}$ to compute optical properties of small crystal cubes by expressing their response in terms of an integral equation involving their surface polarization, which in turn he solved self-consistently by considering a discrete set of points on the surfaces. An equivalent approach, consisting in using a self-consistent relation for the induced surface charge rather than the surface polarization, has been proposed to calculate plasmon excitation frequencies and electron energy losses near arbitrarily shaped interfaces. ${ }^{28}$ This method has been employed to calculate energy losses near metallic spheres lying on planar surfaces $^{29}$ and plasmon frequencies of channels cut into otherwise planar surfaces. ${ }^{33}$ More recently, it has been applied to calculate electron energy losses near other translationally invariant interfaces ${ }^{30-32}$ and axially symmetric interfaces, ${ }^{30}$ taking advantage of the symmetry to reduce the dimension of the problem.

The finiteness of the speed of light $c$ becomes important when the time needed to propagate an electromagnetic signal through the characteristic wavelength $d$ of the charge density fluctuations associated to a given mode of frequency $\omega$ is comparable to its period of oscillation, ${ }^{34}$ that is, when $\omega d / c>1$. For instance, in a sphere of radius $a$, the modes of angular momentum number $l$ are characterized by $l$ nodes in the polar direction, so that $d \sim a / l$ and they suffer important retardation effects when $\omega a / c>l .{ }^{35}$

The electron velocity plays the role of determining which modes are efficiently excited. The electromagnetic field set up by the electron has a characteristic range given by the adiabatic impact parameter $b \sim v / \omega$, so that only modes of comparable or smaller wavelength $d$ can be excited, and therefore, retardation effects require that $v / c \sim 1$ in order to be observed. In addition, when this latter condition is fulfilled, retardation effects appear also in the propagation of the external electromagnetic field set up by the electron, leading to an effective relativistic contraction of the impact parameter. $^{36,17,37,38}$

Cherenkov losses arise in bulk materials when sufficiently large positive values of the real part of the dielectric function lower the velocity of radiation inside the medium below the electron velocity. This type of radiation can also be produced for electrons passing outside the sample, as shown below for electrons moving parallel to dielectric wedges.

The effect of retardation on electron energy loss has been analytically investigated for relativistic electrons traversing foils, ${ }^{39,40}$ or moving near planar surfaces, ${ }^{36,41}$ cylinders, ${ }^{17}$ or spheres. ${ }^{37,38}$ For arbitrary geometries, retardation can be incorporated by using the so-called discrete-dipole approxima- 
tion, also known as coupled-dipole method, ${ }^{42-44}$ first introduced by Purcell and Pennypacker ${ }^{42}$ in the study of optical properties of dielectric grains, and later applied to selfstanding spheres and two neighboring spheres. ${ }^{43}$ This method relies upon intensive numerical calculations, since it basically consists in modeling the sample by an array of dipoles distributed throughout its volume and coupled to each other. It has advantages for very complex geometries and has been applied in its nonretarded version to carbon nanostructures by assigning an induced dipole to describe each carbon atom, with the polarizability adjusted to reproduce the response of graphite. ${ }^{45,46}$ However, this method is essentially three dimensional and becomes prohibitive for large structures.

A method which combines the advantage of being essentially two dimensional with the incorporation of retardation has been developed by Pendry and co-workers to obtain dispersion curves ${ }^{47,48}$ and energy losses ${ }^{49}$ in periodic dielectric arrays. They solved the Maxwell equations by starting from the electric and magnetic fields at a given plane and then propagating these fields to consecutive parallel planes until the entire unit cell of the array was covered, constructing in this way a transfer matrix. Finite difference in the timedomain methods, where spectral properties are obtained by Fourier transforming the electromagnetic field calculated within a time-dependent approach, have been also employed in this context. ${ }^{50}$ However, these methods exhibit slow convergence in systems with large dielectric contrast or thin skin depths. Also, the transfer matrix approach and other methods based upon plane wave ${ }^{51}$ and Bloch wave ${ }^{52}$ expansions are difficult to apply to finite geometries.

Multiple-scattering techniques designed to solve the Maxwell equations for clusters of dielectric objects by reducing the problem to the knowledge of the scattering properties of the individual constituents have been developed for periodic systems of spheres ${ }^{53-56}$ and cylinders, ${ }^{57,58}$ and more recently, generalized to arbitrarily distributed finite clusters of spheres, ${ }^{59}$ with explicit application to electron energy loss spectroscopy (EELS). ${ }^{60}$ But these methods require the scattering matrices of the cluster constituents as input, which are analytical only for certain simple geometries such as cylinders or spheres.

A method for simulating EELS with the incorporation of retardation effects in the presence of arbitrarily shaped interfaces has been recently proposed by the authors. ${ }^{37}$ It consists in expressing the three-dimensional dependence of the scalar and vector potentials in terms of interface charges and currents via a set of surface integral equations. This is the boundary element method ${ }^{61}$ (BEM), where the only assumption, also common to all previously discussed methods, is that the different media involved in the structures under study are described by frequency-dependent local dielectric functions and terminate in abrupt interfaces.

The assumption of isotropic media followed here is generally valid, except in highly anisotropic materials such as multiwall nanotubes and multishell fullerenes. ${ }^{62,45,63,64}$ Moreover, local, frequency-dependent dielectric media are commonly assumed in theoretical studies. This approximation is well justified for aloof geometries or large electron veloci- ties, in which case the effect of dispersion in the response function is usually not important. ${ }^{65}$ The question of the validity of the local response approximation and the neglect of quantum-size effects in small targets has been also addressed $^{66}$ and it has been suggested that sizes above a few nanometers guarantee the absence of this type of effects in metallic systems. ${ }^{66}$

A derivation of the BEM is presented below in Sec. II. The formalism is general enough to be able to deal with magnetic materials as well as with heterostructures formed by an arbitrary number of different materials. The BEM can be used for any external perturbation and the application to the simulation of EELS is discussed in Sec. III. The cases of targets characterized by either translational invariance along one particular direction or axial symmetry are discussed in Secs. IV and V, respectively, where numerical examples are offered. Special emphasis is placed in comparing the results of both nonretarded (Poisson's equation) and fully retarded (Maxwell's equations) calculations. Finally, the main conclusions of this work are summarized in Sec. VI. Gaussian atomic units (a.u., that is, $\hbar=m=e=1$ ) will be used from now on, unless otherwise specified.

\section{BOUNDARY ELEMENT METHOD FOR SOLVING THE MAXWELL EQUATIONS IN INHOMOGENEOUS DIELECTRICS}

The solution of Maxwell's equations in the presence of arbitrarily shaped abrupt dielectric interfaces is expressed in this section in terms of surface integrals involving interface charges and currents that act as sources of the induced electromagnetic field. This is the boundary element method ${ }^{61}$ (BEM), consisting in self-consistently finding those interface distributions for a given external field.

A derivation of the BEM is presented in Sec. II A for the case of interfaces separating two different media. Further details concerning the numerical procedure followed to solve the self-consistent surface-integral equations are given in Sec. II B. The generalization to an arbitrary number of media is offered in Sec. II C. Finally, the nonretarded limit is discussed in Sec. II D. As a guide to this section, a schematic representation of the different relevant elements involved in the theory is shown in Fig. 1.

\section{A. Basic elements of the theory}

Let us start from Maxwell's equations in frequency space $\omega:^{67}$

$$
\begin{gathered}
\nabla \cdot \mathbf{D}=4 \pi \rho, \quad \nabla \times \mathbf{H}+i k \mathbf{D}=\frac{4 \pi}{c} \mathbf{j}, \\
\nabla \cdot \mathbf{B}=0, \quad \nabla \times \mathbf{E}-i k \mathbf{B}=0,
\end{gathered}
$$

where $k=\omega / c, \mathbf{D}=\epsilon \mathbf{E}$ is the electric displacement, and $\mathbf{B}$ $=\mu \mathbf{H}$ is the magnetic induction. In the local response approximation used here, the dielectric function $\epsilon(\mathbf{r}, \omega)$ and the magnetic permeability $\mu(\mathbf{r}, \omega)$ might depend upon space $\mathbf{r}$ and frequency $\omega$. The last two Maxwell equations permit us 


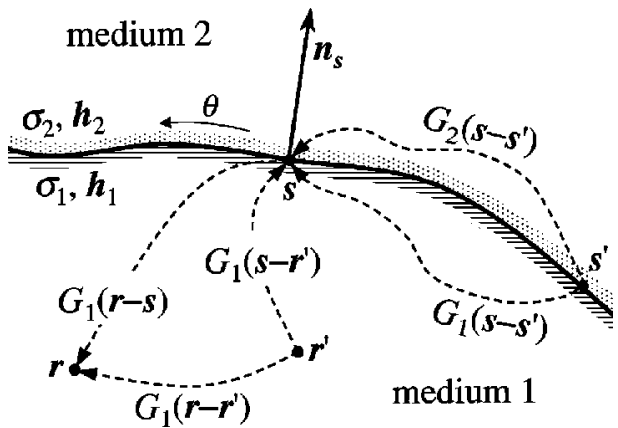

FIG. 1. Schematic representation of the elements involved in the solution of Maxwell's equations in the presence of arbitrarilyshaped sharp interfaces by means of the boundary element method (BEM). The interface shown in the figure (solid curve) separates medium 1 from medium 2. The interface normal $\mathbf{n}_{s}$ at a given interface point $\mathbf{s}$ is chosen to be directed towards medium 2 . The interaction between different points within a given medium $j$ is described by means of the Green function $G_{j}$ [see Eq. (8)], and in particular, the electromagnetic field at $\mathbf{r}$ (within $j=1$ in the figure) is expressed in terms of the external sources (located at $\mathbf{r}^{\prime}$ in the figure) plus some equivalent boundary charges and currents, $\sigma_{j}(\mathbf{s})$ and $\mathbf{h}_{j}(\mathbf{s})$, respectively, which account for the action of any external and induced sources beyond the boundary surface [see Eqs. (6) and (7)]. The fields at $\mathbf{s}$ are also constructed in this way on both sides of the interface and the well-known boundary conditions for the electromagnetic field are satisfied by choosing appropriate values for the equivalent boundary sources.

to express the electric and magnetic fields $\mathbf{E}$ and $\mathbf{H}$ in terms of scalar and vector potentials $\phi$ and $\mathbf{A}$ as

$$
\mathbf{E}=i k \mathbf{A}-\nabla \phi
$$

and

$$
\mathbf{H}=\frac{1}{\mu} \nabla \times \mathbf{A} .
$$

In the spirit of the Lorentz gauge, the convenient relation

$$
\nabla \cdot \mathbf{A}=i k \epsilon \mu \phi
$$

will be adopted here. Then, the first two Maxwell equations can be recast as

$$
\left(\nabla^{2}+k^{2} \epsilon \mu\right) \phi=-4 \pi\left(\frac{\rho}{\epsilon}+\sigma_{s}\right)
$$

and

$$
\left(\nabla^{2}+k^{2} \epsilon \mu\right) \mathbf{A}=-\frac{4 \pi}{c}(\mu \mathbf{j}+\mathbf{m}),
$$

where

$$
\sigma_{s}=\frac{1}{4 \pi} \mathbf{D} \cdot \nabla \frac{1}{\epsilon}
$$

and

$$
\mathbf{m}=-\frac{1}{4 \pi}[i \omega \phi \nabla(\epsilon \mu)+c \mathbf{H} \times \nabla \mu] .
$$

The above expressions remain valid for any arbitrary spatially-dependent dielectric function $\epsilon$ and magnetic permeability $\mu$. Here, we focus on abrupt interfaces that separate two homogeneous media, labeled 1 and 2 , characterized by frequency-dependent response functions $\left(\epsilon_{1}, \mu_{1}\right)$ and $\left(\epsilon_{2}, \mu_{2}\right)$, respectively. ${ }^{68}$

The quantities $\sigma_{s}$ and $\mathbf{m}$ take nonzero values only at the interface, so that they can be understood as additional charges and currents on the dielectric boundary, originating in the discontinuity of $\epsilon$ and $\mu .^{68}$ In particular, the vector $\mathbf{m}$ is not parallel to the interface ${ }^{68}$ and indeed it is normal to the latter in nonmagnetic materials, so that it cannot represent a physical current. However, $\sigma_{s}$ is connected to the interface polarization charges. ${ }^{67}$ Rather than using $\sigma_{s}$ and $\mathbf{m}$, we shall introduce equivalent surface charges and currents in what follows, which will be determined by applying the appropriate boundary conditions for the electromagnetic field in media separated by abrupt interfaces.

The general solution of Eqs. (4) and (5) that vanishes at infinity will be written, for $\mathbf{r}$ inside each medium $j$, as

$$
\begin{gathered}
\phi(\mathbf{r})=\frac{1}{\epsilon_{j}(\omega)} \int d \mathbf{r}^{\prime} G_{j}\left(\left|\mathbf{r}-\mathbf{r}^{\prime}\right|\right) \rho\left(\mathbf{r}^{\prime}\right) \\
+\int_{S_{j}} d \mathbf{s} G_{j}(|\mathbf{r}-\mathbf{s}|) \sigma_{j}(\mathbf{s})
\end{gathered}
$$

and

$$
\begin{aligned}
\mathbf{A}(\mathbf{r})= & \frac{\mu_{j}(\omega)}{c} \int d \mathbf{r}^{\prime} G_{j}\left(\left|\mathbf{r}-\mathbf{r}^{\prime}\right|\right) \mathbf{j}\left(\mathbf{r}^{\prime}\right) \\
& +\int_{S_{j}} d \mathbf{s} G_{j}(|\mathbf{r}-\mathbf{s}|) \mathbf{h}_{j}(\mathbf{s}),
\end{aligned}
$$

where $S_{j}$ refers to the boundary of medium $j=1,2$;

$$
G_{j}(r)=\frac{e^{i k_{j} r}}{r}
$$

is the Green function of the wave equation

$$
\left[\nabla^{2}+k_{j}^{2}\right] G_{j}(r)=-4 \pi \delta(\mathbf{r})
$$

in medium $j ; k_{j}=k \sqrt{\epsilon_{j} \mu_{j}}$; and the square root is understood to yield positive imaginary parts (this sign choice ensures that $\phi$ and $\mathbf{A}$ actually vanish at infinity, while at the same time, it is consistent with the retarded response formalism adopted in this paper). That Eqs. (6) and (7) are indeed the solution of Eqs. (4) and (5) can be seen upon inspection: by construction, the first integrals on the right hand side of Eqs. (6) and (7) satisfy Eqs. (4) and (5) outside the interfaces, where $\sigma_{s}=0$ and $\mathbf{m}=0 .{ }^{68}$ The additional surface integrals are required partly to include the effects of $\sigma_{s}$ and $\mathbf{m}$ and partly to compensate for the fact that the ad hoc Green function $G\left(\left|\mathbf{r}-\mathbf{r}^{\prime}\right|\right)=G_{j}\left(\left|\mathbf{r}-\mathbf{r}^{\prime}\right|\right)$ for $\mathbf{r}$ lying in medium $j$ is discontinuous at the interfaces. ${ }^{69}$ Therefore, Eqs. (6) and (7) are the solution of Eqs. (4) and (5) provided one can choose the boundary charges and currents $\sigma_{j}$ and $\mathbf{h}_{j}$ in such a way that the resulting electromagnetic field satisfies the customary boundary conditions. ${ }^{67,69}$ Notice that $\sigma_{j}$ and $\mathbf{h}_{j}$ do not gen- 
erally represent real interface charges. ${ }^{70}$ However, in the nonretarded limit $(c \rightarrow \infty), \sigma_{1}$ can be made to coincide with $\sigma_{2}$ and represents a physical induced interface charge, as shown below in Sec. II D.

The continuity of the tangential electric field and the normal magnetic induction at the interface, together with the gauge condition given by Eq. (3), leads to the continuity of $\phi$ and $\mathbf{A}$ [see Eqs. (1) and (2)]. That is, Eqs. (6) and (7) have to have the same value on either side of the interface that separates medium 1 from medium 2, and therefore, one has

$$
G_{1} \sigma_{1}-G_{2} \sigma_{2}=\phi_{2}^{e}-\phi_{1}^{e}
$$

and

$$
G_{1} \mathbf{h}_{1}-G_{2} \mathbf{h}_{2}=\mathbf{A}_{2}^{e}-\mathbf{A}_{1}^{e},
$$

respectively, where

$$
\phi_{j}^{e}(\mathbf{s})=\frac{1}{\epsilon_{j}(\omega)} \int d \mathbf{r}^{\prime} G_{j}\left(\left|\mathbf{s}-\mathbf{r}^{\prime}\right|\right) \rho\left(\mathbf{r}^{\prime}\right)
$$

and

$$
\mathbf{A}_{j}^{e}(\mathbf{s})=\frac{\mu_{j}(\omega)}{c} \int d \mathbf{r}^{\prime} G_{j}\left(\left|\mathbf{s}-\mathbf{r}^{\prime}\right|\right) \mathbf{j}\left(\mathbf{r}^{\prime}\right)
$$

are equivalent boundary sources that scale linearly with the external perturbation. Matrix notation has been adopted in Eqs. (10) and (11), so that interface coordinates $\mathbf{s}$ are used as matrix and vector indices, and matrix-vector products such as $G_{j} \sigma_{j}$ involve integration over the interface.

The equivalent boundary sources $\phi_{j}^{e}$ and $\mathbf{A}_{j}^{e}$ are the scalar and vector potentials that would be created at the interface points by the external charges and currents if the entire space were homogeneous and filled with medium $j$. Accordingly, the right hand side of Eqs. (10) and (11) represent the jump in such potentials between $j=1$ and $j=2$.

In the nonmagnetic case (i.e., when $\mu_{j}=1$ ), the continuity of the tangential magnetic field and the vector potential implies that both the tangential derivatives of all components of the vector potential and the normal derivative of the tangential vector potential must be continuous [see Eq. (2)]. Using this and Eq. (3), one has that $\left(\mathbf{n}_{s} \cdot \nabla\right) \mathbf{A}-i \mathbf{n}_{s} k \epsilon \mu \phi$ is continuous as well. Inserting Eqs. (6) and (7) into this last expression, one finds

$$
H_{1} \mathbf{h}_{1}-H_{2} \mathbf{h}_{2}-i k \mathbf{n}_{s}\left(G_{1} \epsilon_{1} \mu_{1} \sigma_{1}-G_{2} \epsilon_{2} \mu_{2} \sigma_{2}\right)=\vec{\alpha}
$$

( $\mu_{j}$ is maintained here for later reference), where

$$
\vec{\alpha}=\left(\mathbf{n}_{s} \cdot \nabla_{\mathbf{s}}\right)\left(\mathbf{A}_{2}^{e}-\mathbf{A}_{1}^{e}\right)+i k \mathbf{n}_{s}\left(\epsilon_{1} \mu_{1} \phi_{1}^{e}-\epsilon_{2} \mu_{2} \phi_{2}^{e}\right)
$$

and $H_{j}$ is the normal derivative of the Green function $G_{j}$, which must be taken from both sides of the interface. More precisely,

$$
\begin{aligned}
H_{1}\left(\mathbf{s}, \mathbf{s}^{\prime}\right) & =\lim _{\eta \rightarrow 0^{+}} \mathbf{n}_{s} \cdot \nabla_{\mathbf{s}} G_{1}\left(\left|\mathbf{s}-\eta \mathbf{n}_{s}-\mathbf{s}^{\prime}\right|\right) \\
& =\mathbf{n}_{s} \cdot \nabla_{\mathbf{s}} G_{1}\left(\left|\mathbf{s}-\mathbf{s}^{\prime}\right|\right)+2 \pi \delta\left(\mathbf{s}-\mathbf{s}^{\prime}\right)
\end{aligned}
$$

and

$$
\begin{aligned}
H_{2}\left(\mathbf{s}, \mathbf{s}^{\prime}\right) & =\lim _{\eta \rightarrow 0^{+}} \mathbf{n}_{s} \cdot \nabla_{\mathbf{s}} G_{2}\left(\left|\mathbf{s}+\eta \mathbf{n}_{s}-\mathbf{s}^{\prime}\right|\right) \\
& =\mathbf{n}_{s} \cdot \nabla_{\mathbf{s}} G_{2}\left(\left|\mathbf{s}-\mathbf{s}^{\prime}\right|\right)-2 \pi \delta\left(\mathbf{s}-\mathbf{s}^{\prime}\right),
\end{aligned}
$$

where

$$
\mathbf{n}_{s} \cdot \nabla_{\mathbf{s}} G_{j}\left(\left|\mathbf{s}-\mathbf{s}^{\prime}\right|\right)=\frac{\mathbf{n}_{s} \cdot\left(\mathbf{s}-\mathbf{s}^{\prime}\right)}{\left|\mathbf{s}-\mathbf{s}^{\prime}\right|^{3}}\left(i k_{j}\left|\mathbf{s}-\mathbf{s}^{\prime}\right|-1\right) e^{i k_{j}\left|\mathbf{s}-\mathbf{s}^{\prime}\right|},
$$

Eq. (A2) of Appendix A has been employed, and the interface normal $\mathbf{n}_{s}$ has been chosen to be directed towards medium 2 (see Fig. 1). In a homogeneously charged infinite plane, the $\delta$ functions in $H_{j}$ account for the normal electric field of opposite direction on either side of the plane.

For magnetic materials, the projection of Eq. (14) on $\mathbf{n}_{s}$ still holds as a result of Eq. (3), but the tangential part must be replaced by

$$
\begin{gathered}
\left(\mathbf{n}_{s} \partial_{i} G_{1}-\mathbf{t}_{s}^{i} H_{1}\right) \frac{1}{\mu_{1}} \cdot \mathbf{h}_{1}-\left(\mathbf{n}_{s} \partial_{i} G_{2}-\mathbf{t}_{s}^{i} H_{2}\right) \frac{1}{\mu_{2}} \cdot \mathbf{h}_{2} \\
=\left[\mathbf{n}_{s} \cdot \partial_{i}-\mathbf{t}_{s}^{i} \cdot\left(\mathbf{n}_{s} \cdot \nabla_{\mathbf{s}}\right)\right]\left(\frac{\mathbf{A}_{2}^{e}}{\mu_{2}}-\frac{\mathbf{A}_{1}^{e}}{\mu_{1}}\right), \quad i=1,2,
\end{gathered}
$$

where $\mathbf{t}_{s}^{1}$ and $\mathbf{t}_{s}^{2}$ are two independent tangential vectors and the derivative $\partial_{i}$ is taken along the direction $\mathbf{t}_{s}^{i}$. Eq. (16) comes from the continuity of the tangential magnetic field.

Finally, the continuity of the normal electric displacement $\epsilon \mathbf{n}_{s} \cdot(i k \mathbf{A}-\nabla \phi)$, leads to

$$
H_{1} \epsilon_{1} \sigma_{1}-H_{2} \epsilon_{2} \sigma_{2}-i k \mathbf{n}_{s} \cdot\left(G_{1} \epsilon_{1} \mathbf{h}_{1}-G_{2} \epsilon_{2} \mathbf{h}_{2}\right)=D^{e},
$$

where

$$
D^{e}=\mathbf{n}_{s} \cdot\left[\epsilon_{1}\left(i k \mathbf{A}_{1}^{e}-\nabla_{\mathbf{s}} \phi_{1}^{e}\right)-\epsilon_{2}\left(i k \mathbf{A}_{2}^{e}-\nabla_{\mathbf{s}} \phi_{2}^{e}\right)\right]
$$

is the difference of the normal displacement that would be created at the interface by the external sources if the entire space were homogeneous and filled with either medium $j$ $=2$ or medium $j=1$, respectively.

In brief, it has been shown that when the auxiliary boundary charges and currents $\sigma_{j}$ and $\mathbf{h}_{j}$ are self-consistently calculated from Eqs. (10), (11), (14), and (17) then Eqs. (6) and (7) constitute the solution of the Maxwell equations that vanishes at infinity and satisfies appropriate boundary conditions at the interfaces. For magnetic materials, the projection of Eq. (14) on the interface directions has to be replaced by Eq. (16). The external source is introduced via inhomogeneous terms, as defined by Eqs. (12), (13), (15), and (18). Magnetic media will not be considered here, although they can be dealt with by straightforward generalization of what follows.

\section{B. Numerical procedure}

Taking into account the vectorial character of some of the quantities involved in Eqs. (10), (11), (14), and (17), one can realize that these constitute a system of eight linear surfaceintegral equations with eight unknown complex functions of the interface coordinates (namely, $\sigma_{j}$ and $\mathbf{h}_{j}$ for $j=1,2$ ). Different methods can be employed to solve this system of 
equations. For instance, in simple geometries, one can project the interface sources, charges, and currents on a truncated basis set (e.g., Bessel functions in the case of axially symmetric systems). Instead, we have chosen a method that can be straightforwardly applied to arbitrary geometries. It consists in discretizing the interface integrals, that is, in reducing the spatial dependence of each quantity to a discrete number $N$ of representative points distributed along the interface $\left(\mathbf{s}_{a}\right.$, for $\left.a=1, \ldots, N\right)$. Associated to each of these points, we will consider an interface element $\delta S_{a}$ such that this defines a perfect tiling of the interfaces.

Both the external field and the unknowns $\left(\sigma_{j}, \mathbf{h}_{j}\right)$ will be assumed to exhibit only very small variations between contiguous points of the discretization, so that they can be considered to be nearly constant within each interface element. This is certainly the case when a sufficiently large value of $N$ is taken and the external charge and current distributions do not overlap the interface. Potential problems at sharp edges and corners are effectively prevented by rounding them off, which is justified on physical grounds.

With this discretization, the operators that appear in the noted system of equations can be approximated by finite matrices of dimension $N \times N$. Besides, the interface charges and currents are approximated by complex vectors of dimension $N$, containing the value of $\left(\sigma_{j}, \mathbf{h}_{j}\right)$ at the $N$ representative points. Notice that both $H_{j}\left(\mathbf{s}, \mathbf{s}^{\prime}\right)$ and $G_{j}\left(\left|\mathbf{s}-\mathbf{s}^{\prime}\right|\right)$ diverge in the $\mathbf{s} \rightarrow \mathbf{s}^{\prime}$ limit. However, this is an integrable divergence that is easily overcome by averaging over the small interface elements. More explicitly, the components of matrix $G_{j}$ and vector $\sigma_{j}$ read

$$
G_{j, a b}=\int_{\delta S_{b}} d \mathbf{s}^{\prime} G_{j}\left(\left|\mathbf{s}_{a}-\mathbf{s}^{\prime}\right|\right)
$$

and

$$
\sigma_{j, a}=\sigma_{j}\left(\mathbf{s}_{a}\right),
$$

where the indices $a$ and $b$ label interfaces elements. One can proceed with other quantities in a similar way. For instance, $\delta\left(\mathbf{s}-\mathbf{s}^{\prime}\right)$ becomes $\delta_{a b}$, and $\mathbf{n}_{s}$ and $\epsilon_{j}$ must be interpreted as diagonal matrices with elements $\mathbf{n}_{s_{a}} \delta_{a b}$ and $\epsilon_{j} \delta_{a b}$.

The resulting discretized system involves $8 N$ linear equations and $8 N$ complex variables, which require a computation time proportional to $(8 N)^{3}$ to be solved by direct inversion. A more efficient procedure is followed here by separately manipulating matrices of dimension $N \times N$ such as $G_{j}$. After some algebraic manipulations, the solution of Eqs. (10), (11), (14), and (17) is found to be, for nonmagnetic materials $\left(\mu_{j}=1\right)$,

$$
\begin{aligned}
\sigma_{j}= & G_{j}^{-1} \Sigma^{-1}\left\{D^{e}-\epsilon_{j^{\prime}} \Sigma_{j^{\prime}} \phi^{e}+i k \mathbf{n}_{s} \cdot \Delta^{-1}\left[\left(\epsilon_{1}-\epsilon_{2}\right)(\vec{\alpha}\right.\right. \\
& \left.\left.\left.+i k \mathbf{n}_{s} \epsilon_{j^{\prime}} \phi^{e}\right)+\left(\epsilon_{2} \Sigma_{1}-\epsilon_{1} \Sigma_{2}\right) \mathbf{A}^{e}\right]\right\}
\end{aligned}
$$

and

$$
\mathbf{h}_{j}=G_{j}^{-1} \Delta^{-1}\left[\vec{\alpha}-\Sigma_{j^{\prime}} \mathbf{A}^{e}+i k \mathbf{n}_{s}\left(G_{1} \epsilon_{1} \sigma_{1}-G_{2} \epsilon_{2} \sigma_{2}\right)\right],
$$

where

$$
\begin{gathered}
\phi^{e}=\phi_{2}^{e}-\phi_{1}^{e}, \quad \mathbf{A}^{e}=\mathbf{A}_{2}^{e}-\mathbf{A}_{1}^{e}, \\
\Sigma_{j}=H_{j} G_{j}^{-1}, \\
\Delta=\Sigma_{1}-\Sigma_{2}, \\
\Sigma=\epsilon_{1} \Sigma_{1}-\epsilon_{2} \Sigma_{2}+k^{2}\left(\epsilon_{1}-\epsilon_{2}\right)^{2} \mathbf{n}_{s} \cdot \Delta^{-1} \mathbf{n}_{s},
\end{gathered}
$$

and $j^{\prime}=2,1$ when $j=1,2$. Just to make the notation clearer, the $(a b)$ component of matrix $\mathbf{n}_{s} \cdot \Delta^{-1} \mathbf{n}_{s}$ in Eq. (21) reads $\left(\mathbf{n}_{s_{a}} \cdot \mathbf{n}_{s_{b}}\right)\left[\Delta^{-1}\right]_{a b}$.

Equations (19), (20), and (21) are actually a powerful tool that can be used to find analytical solutions of Maxwell's equations in simple geometries (e.g., planes, spheres, cylinders, or spheroids) by projecting matrices and vectors on a basis of analytical functions (e.g., plane waves or Bessel functions). Previous analytical results for the cylinder ${ }^{17}$ and the sphere ${ }^{38}$ are readily reproduced.

The natural oscillation modes of the system are also obtained from the relation $\operatorname{det}\{\Sigma\}=0$. For instance, in a planar interface, working in momentum space $\mathbf{Q}$ along the interface directions, one has $G_{j}=2 \pi / \sqrt{Q^{2}-k_{j}^{2}}, H_{1}=2 \pi$, and $H_{2}=$ $-2 \pi$, and upon substitution in Eq. (21), one finds the wellknown dispersion relation ${ }^{71} Q=k\left[\epsilon_{1} \epsilon_{2} /\left(\epsilon_{1}+\epsilon_{2}\right)\right]^{1 / 2}$, which in the nonretarded limit reduces to $\epsilon_{1}+\epsilon_{2}=0$.

Equations (19), (20), and (21) can be evaluated with only four matrix inversions and two matrix multiplications, if one neglects addition of matrices, multiplication by diagonal matrices, and multiplication of matrices by vectors, which require a computation time proportional to $N^{2}$. Thus, the computational cost of evaluating these equations is proportional to $6 N^{3}$.

\section{Generalization to an arbitrary number of media}

The formalism presented in Secs. II A and II B can be generalized to geometries that involve more than two different materials by performing a few redefinitions. First of all, the Green function $G_{j}$ will be taken to be given by Eq. (8) only when it connects points on the inner interface of a homogeneous region of space filled with medium $j$ and it will be set to zero otherwise. Moreover, $\left(\epsilon_{2}, \mu_{2}\right)$ and $\left(\epsilon_{1}, \mu_{1}\right)$ will be now taken to represent the response functions of the materials $j_{2}$ and $j_{1}$ that lie on the side pointed to by the interface normal and on the opposite side, respectively. Naturally, these variables, including $j_{1}$ and $j_{2}$, might depend on the interface coordinate $\mathbf{s}_{a}$. This dependence will be denoted $\epsilon_{1, a}=\epsilon_{j_{1}(a)}$, and so on. (Notice that the interface normals cannot be chosen in general such that those surrounding every homogeneous region of space point either all inside or all outside that region.)

Using the matrix notation introduced in last section, the component $a$ of terms such as $H_{1} \epsilon_{1} \sigma_{1}-H_{2} \epsilon_{2} \sigma_{2}$ in Eq. (17) has to be interpreted as 


$$
\begin{aligned}
\sum_{b}\{ & H_{j_{1}(a), a b} \epsilon_{1, a}\left[\sigma_{1, b} \delta_{j_{1}(a) j_{1}(b)}+\sigma_{2, b} \delta_{j_{1}(a) j_{2}(b)}\right] \\
& \left.-H_{j_{2}(a), a b} \epsilon_{2, a}\left[\sigma_{1, b} \delta_{j_{2}(a) j_{1}(b)}+\sigma_{2, b} \delta_{j_{2}(a) j_{2}(b)}\right]\right\}, \\
= & \sum_{b}\left\{H_{j_{1}(b), a b}\left[\delta_{j_{1}(a) j_{1}(b)}-\delta_{j_{2}(a) j_{1}(b)}\right] \epsilon_{1, b} \sigma_{1, b}\right. \\
& \left.-H_{j_{2}(b), a b}\left[\delta_{j_{2}(a) j_{2}(b)}-\delta_{j_{1}(a) j_{2}(b)}\right] \epsilon_{2, b} \sigma_{2, b}\right\} .
\end{aligned}
$$

This equation expresses the fact that charges that cannot be connected without crossing any interface are blind to each other. ${ }^{69}$ Therefore, Eqs. (10), (11), (14), (16), and (17) remain valid if one makes the substitutions

$$
\begin{aligned}
& G_{1, a b} \leftarrow G_{j_{1}(b), a b}\left[\delta_{j_{1}(a) j_{1}(b)}-\delta_{j_{2}(a) j_{1}(b)}\right], \\
& G_{2, a b} \leftarrow G_{j_{2}(b), a b}\left[\delta_{j_{2}(a) j_{2}(b)}-\delta_{j_{1}(a) j_{2}(b)}\right], \\
& H_{1, a b} \leftarrow H_{j_{1}(b), a b}\left[\delta_{j_{1}(a) j_{1}(b)}-\delta_{j_{2}(a) j_{1}(b)}\right],
\end{aligned}
$$

and

$$
H_{2, a b} \leftarrow H_{j_{2}(b), a b}\left[\delta_{j_{2}(a) j_{2}(b)}-\delta_{j_{1}(a) j_{2}(b)}\right],
$$

where the Green functions on the right hand side of these expressions are calculated with the definition given in Eq. (8).

With these substitutions the boundary currents satisfy still Eq. (20), whereas the boundary charges are resolved as

$$
\begin{aligned}
\sigma_{j}= & G_{j}^{-1} \Sigma^{-1}\left\{D^{e}-\Sigma_{j^{\prime}} L_{j^{\prime}} \phi^{e}+i k \mathbf{n}_{s} \cdot\left[\left(L_{1}-L_{2}\right) \Delta^{-1}(\vec{\alpha}\right.\right. \\
& \left.\left.\left.+i k \mathbf{n}_{s} L_{j^{\prime}} \phi^{e}\right)+\left(L_{2} \Delta^{-1} \Sigma_{1}-L_{1} \Delta^{-1} \Sigma_{2}\right) \mathbf{A}^{e}\right]\right\},
\end{aligned}
$$

where

$$
L_{j}=G_{j} \epsilon_{j} G_{j}^{-1}
$$

and $\Sigma$ needs to be redefined as

$$
\Sigma=\Sigma_{1} L_{1}-\Sigma_{2} L_{2}+k^{2} \mathbf{n}_{s} \cdot\left(L_{1}-L_{2}\right) \Delta^{-1} \mathbf{n}_{s}\left(L_{1}-L_{2}\right) .
$$

Notice that the parametrization can be chosen such that $L_{j}$ $=\epsilon_{j}$ in the two-media case.

\section{Nonretarded limit}

In the nonretarded limit, $k \rightarrow 0$, the Green function (8) reduces to the nonretarded Coulomb interaction $1 / r$. With a suitable redefinition of the external sources (see Ref. 69) $\phi_{j}^{e}$ becomes independent of $j$ [see Eq. (12)]. Furthermore, $\mathbf{A}_{j}^{e}$ and $\vec{\alpha}$ vanish and $D^{e}=\left(\epsilon_{2}-\epsilon_{1}\right) f$ [see Eqs. (15) and (18)], where

$$
f(\mathbf{s})=\int d \mathbf{r}^{\prime} F\left(\mathbf{s}, \mathbf{r}^{\prime}\right) \frac{\rho\left(\mathbf{r}^{\prime}\right)}{\epsilon\left(\mathbf{r}^{\prime}, \omega\right)}
$$

is the external electric field normal to the interface and

$$
F\left(\mathbf{s}, \mathbf{s}^{\prime}\right)=-\frac{\mathbf{n}_{s} \cdot\left(\mathbf{s}-\mathbf{s}^{\prime}\right)}{\left|\mathbf{s}-\mathbf{s}^{\prime}\right|^{3}} .
$$

Eqs. (10), (11), and (14) are trivially satisfied by setting $\mathbf{h}_{j}$ $=0$ and $\sigma_{1}=\sigma_{2}=\sigma$. The remaining Eq. (17) can be used to obtain self-consistently the induced interface charge $\sigma$. One finds (for only two different materials)

$$
\Lambda \sigma(\mathbf{s})=f(\mathbf{s})+\int_{S} d \mathbf{s}^{\prime} F\left(\mathbf{s}, \mathbf{s}^{\prime}\right) \sigma\left(\mathbf{s}^{\prime}\right),
$$

where

$$
\Lambda=2 \pi \frac{\epsilon_{2}+\epsilon_{1}}{\epsilon_{2}-\epsilon_{1}} .
$$

Finally, the induced potential is given in terms of the induced interface charge as ${ }^{69}$

$$
\phi^{\text {ind }}(\mathbf{r})=\int d \mathbf{s} \frac{\sigma(\mathbf{r})}{|\mathbf{r}-\mathbf{s}|} .
$$

Similar expressions were proposed by Fuchs ${ }^{27}$ to obtain surface oscillation modes of complex geometries, and more recently by $\mathrm{Lu}$ and Maradudin ${ }^{33}$ to calculate plasmon modes of channels cut into planar surfaces.

Equation (23) separates the response functions of the two materials that form the structure under consideration (fully contained inside $\Lambda$ ) from the actual geometry of the interface (fully contained on the right hand side of that equation). In the absence of a source, $f=0$ and Eq. (23) reduces to an eigenvalue problem for $\Lambda$. This fact has been used to formulate sum rules that relate the plasmon modes of a given geometry with those of its complementary ${ }^{72}$ (notice that $\Lambda$ changes its sign upon exchange of $\epsilon_{1}$ and $\epsilon_{2}$ ).

Electron energy loss spectra can be simulated by setting the external charge to that of a fast electron. ${ }^{28}$ This method has been applied to electrons passing near spheres lying on planar surfaces, ${ }^{29}$ and more recently, to both translationally invariant interfaces ${ }^{30-32}$ and axially symmetric interfaces ${ }^{30}$ of otherwise arbitrary shape. It has been also shown ${ }^{30}$ that, given a certain interface geometry, the EELS spectrum needs to be calculated only once for a given combination of $\epsilon_{1}$ and $\epsilon_{2}$, so that the result for any other combination can be expressed in terms of that one. Unfortunately, this convenient property does not apply to the retarded case, due to the involved dependence of the Green function (8) on $\epsilon_{j}$. In the present work, nonretarded results for EELS have been obtained using this approach and compared with the retarded calculations offered below.

\section{APPLICATION TO ELECTRON ENERGY LOSS SPECTROSCOPY}

The energy loss suffered by a fast electron passing near an inhomogeneous sample and moving with constant velocity $\mathbf{v}$ along a straight line trajectory described by $\mathbf{r}_{e}(t)$ can be related to the force exerted by the induced electric field $\mathbf{E}^{\text {ind }}$ acting back on the electron as

$$
\Delta E=\int d t \mathbf{v} \cdot \mathbf{E}^{\text {ind }}\left[\mathbf{r}_{e}(t), t\right]=\int_{0}^{\infty} \omega d \omega \Gamma(\omega)
$$


(the -1 electron charge has been included here, so that $\Delta E>0$ with this definition), where

$$
\Gamma(\omega)=\frac{1}{\pi \omega} \int d t \operatorname{Re}\left\{e^{-i \omega t} \mathbf{v} \cdot \mathbf{E}^{\text {ind }}\left[\mathbf{r}_{e}(t), \omega\right]\right\}
$$

is the loss probability. The Fourier transform

$$
\mathbf{E}^{\text {ind }}(\mathbf{r}, t)=\int \frac{d \omega}{2 \pi} e^{-i \omega t} \mathbf{E}^{\text {ind }}(\mathbf{r}, \omega)
$$

has been introduced and the property $\mathbf{E}^{\text {ind }}(\mathbf{r}, \omega)=\left[\mathbf{E}^{\text {ind }}(\mathbf{r}\right.$, $-\omega)]^{*}$ has been used.

The formalism presented here applies to electron trajectories that may cross in general some of the interfaces. The external electron source enters via the equivalent boundary sources $\phi_{j}^{e}, \mathbf{A}_{j}^{e}, \vec{\alpha}$, and $D^{e}$ [Eqs. (12), (13), (15), and (18)].

Fortunately, only electrons moving fully inside homogeneous media need to be considered to obtain the boundary sources. ${ }^{69}$ Writing the electron trajectory as $\mathbf{r}_{e}(t)=\mathbf{b}+\mathbf{v} t$, one obtains analytical expressions for the boundary sources. From Eq. (12), one finds

$$
\phi_{j}^{e}(\mathbf{s})=\frac{-2}{v \epsilon_{j}} K_{0}\left(\frac{\omega\left|\mathbf{s}^{\perp}-\mathbf{b}^{\perp}\right|}{v \gamma_{j}}\right) e^{i \omega\left(s^{\|}-b^{\|}\right) / v},
$$

where $\gamma_{j}=1 / \sqrt{1-\epsilon_{j} \mu_{j} v^{2} / c^{2}}, \quad K_{0}$ is the modified Bessel function of order 0 , and the symbol $\|(\perp)$ refers to components parallel (perpendicular) with respect to the velocity vector v. Also, Eq. (13) permits one to write

$$
\mathbf{A}_{j}^{e}(\mathbf{s})=\epsilon_{j} \mu_{j} \frac{\mathbf{v}}{c} \phi_{j}^{e}(\mathbf{s})
$$

Finally, $\vec{\alpha}$ and $D^{e}$ are easily obtained by inserting these expressions into Eqs. (15) and (18), respectively.

The contribution to the loss probability coming from the first terms in Eqs. (6) and (7) does not contain any information about the interface, and it is the same as if the electron were traveling inside the bulk of an infinite material described by a dielectric function $\epsilon_{j}$ and a magnetic permeability $\mu_{j}$. This permits one to separate the loss probability $\Gamma$ as

$$
\Gamma=\Gamma^{\text {bulk }}+\Gamma^{\text {boundary }},
$$

where

$$
\Gamma^{\text {bulk }}(\omega)=\sum_{j} \frac{L_{j}}{\pi v^{2}} \operatorname{Im}\left\{\left(\frac{v^{2}}{c^{2}} \mu_{j}-\frac{1}{\epsilon_{j}}\right) \ln \left[\frac{q_{c}^{2}-k_{j}^{2}}{(\omega / v)^{2}-k_{j}^{2}}\right]\right\}
$$

corresponds to the contribution just noted. Here, $k_{j}^{2}$ $=(\omega / c)^{2} \epsilon_{j} \mu_{j}, L_{j}$ is the length of the trajectory inside me$\operatorname{dium} j$, and $q_{c}$ is a momentum cut-off that, in the absence of a microscope aperture limiting the electron scattering angles, is determined by energy conservation.

When $\epsilon_{j}$ and $\mu_{j}$ are real (except for a positive infinitesimal imaginary part), bulk Cherenkov losses arising from the denominator of the ln argument of Eq. (26) can be clearly identified as ${ }^{67}$

$$
\Gamma_{\text {Cherenkov }}^{\text {bulk }}(\omega)=\left|\mu_{j}\right| L_{j}\left(\frac{1}{c^{2}}-\frac{1}{v^{2} \epsilon_{j} \mu_{j}}\right),
$$

subject to the Cherenkov condition $v^{2} \epsilon_{j} \mu_{j}>c^{2}$. Equations (26) and (27) are also valid for left-handed materials, ${ }^{73}$ where the real part of $\epsilon_{j}$ and $\mu_{j}$ are simultaneously negative.

The remaining contribution $\Gamma^{\text {boundary }}$ comes entirely from the induced electric field produced by the auxiliary boundary charges and currents [the second terms in Eqs. (6) and (7)]. $\Gamma^{\text {boundary }}$ results in characteristic energy losses that are related to the geometry of the interface.

Inserting Eqs. (6) and (7) into Eq. (1) to calculate the electric field, one obtains

$$
\begin{aligned}
\Gamma^{\text {boundary }}(\omega)= & \frac{1}{\pi \omega} \sum_{j} \int_{t_{j}^{0}}^{t_{j}^{1}} d t \operatorname{Re}\left\{e^{-i \omega t}\right. \\
& \times \int_{S_{j}} d \mathbf{s v} \cdot\left[i k G_{j}\left(\left|\mathbf{r}_{e}-\mathbf{s}\right|\right) \mathbf{h}_{j}(\mathbf{s})\right. \\
& \left.\left.-\nabla_{\mathbf{r}_{e}} G_{j}\left(\left|\mathbf{r}_{e}-\mathbf{s}\right|\right) \sigma_{j}(\mathbf{s})\right]\right\}
\end{aligned}
$$

where the time integral is extended over the part of the trajectory inside each medium $j$ visited by the electron.

Equation (28) is general and it can be used to derive analytical expressions for the energy loss probability in simple geometries such as the planar surface, ${ }^{36,41}$ the cylinder, ${ }^{17}$ or the sphere. ${ }^{37,38}$ However, we are concerned here with more complex interfaces, which cannot be worked out analytically. In particular, the cases of translationally-invariant interfaces and axially symmetric interfaces will be discussed in Secs. IV and V.

\section{EELS IN TRANSLATIONALLY INVARIANT INTERFACES}

When one considers interfaces that are invariant under translations along one particular direction, but otherwise of arbitrary shape, the number of points needed to evaluate the auxiliary boundary charges and currents given by Eqs. (19), (20), and (22) is considerably reduced by working in the space of Fourier transform along the direction of symmetry.

For invariance under translations along the $z$ axis, the interfaces can be parametrized as $\mathbf{s}(\theta, z)=\left[\mathbf{R}_{s}(\theta), z\right]$, where $\mathbf{R}_{s}(\theta)=\left[x_{s}(\theta), y_{s}(\theta)\right]$, so that the interface normal $\mathbf{n}_{s}$ $=\left(y_{s}^{\prime},-x_{s}^{\prime}, 0\right) / \sqrt{x_{s}^{\prime 2}+y_{s}^{\prime 2}}$ points towards the medium on the right hand side when one runs along the interface profile in the sense of increasing $\theta$ (see Fig. 1).

Using the convolution theorem, the results of the previous sections can be fully applied by expressing the different quantities in Fourier space with respect to the direction of symmetry. In particular, the boundary charges can be written as

$$
\sigma_{j}(\theta, z)=\int \frac{d q}{2 \pi} \sigma_{j}(\theta, q) e^{i q z}
$$

and the transform of the Green function (8) reduces to 


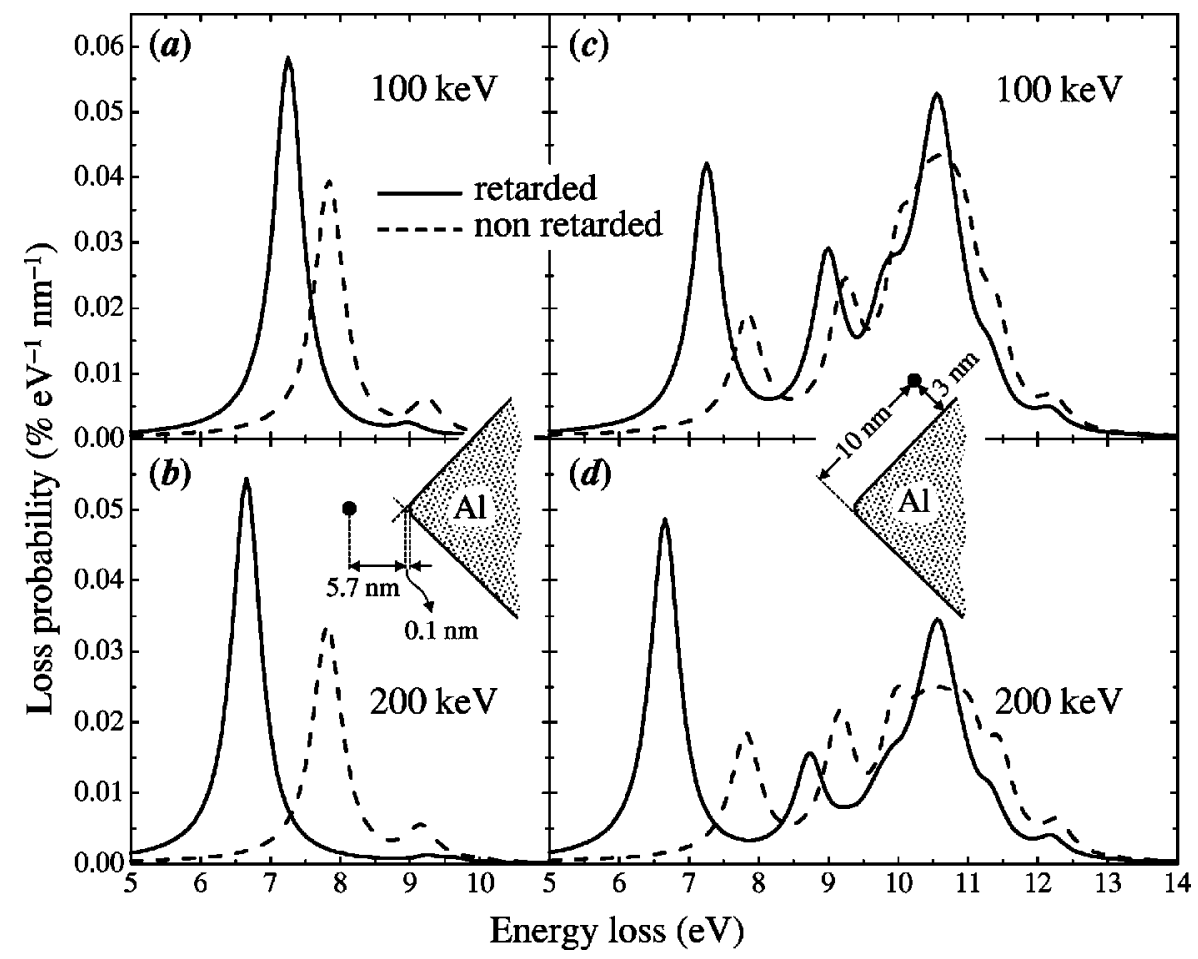

FIG. 2. Energy loss probability per energy range and unit path length for electrons moving parallel to a hyperbolic $90^{\circ} \mathrm{Al}$ wedge. $\mathrm{A}$ section of the wedge is shown in the insets, with the trajectories under discussion indicated by dots (the electrons move perpendicularly to the plane of the paper) and passing close to the wedge corner in (a) and (b) and close to one of the wedge sides in (c) and (d). Retarded (non-retarded) results are shown by solid (broken) curves. Two different electron energies are considered: $100 \mathrm{keV}$ in (a) and (c), and 200 $\mathrm{keV}$ in (b) and (d).

$$
G_{j}(\mathbf{R}, q)=2 K_{0}\left(\frac{q R}{\gamma_{j}}\right),
$$

where $\mathbf{R}$ denotes space coordinates normal to $z, \gamma_{j}$ $=1 / \sqrt{1-k_{j}^{2} / q^{2}}$ and the square root is chosen to yield positive real parts. The surface integrals can be performed making the substitution ${ }^{30}$

$$
\int_{S} \rightarrow \int d \theta J(\theta)
$$

where $J(\theta) d \theta=\sqrt{x_{s}^{\prime}(\theta)^{2}+y_{s}^{\prime}(\theta)^{2}} d \theta$ is an element of length in the contour shown in Fig. 1. Moreover, the integral over $z$ becomes a simple product in Fourier space $q$ as a result of the convolution theorem.

If $k_{j}$ is real and satisfies $k_{j}>q$ then $\gamma_{j}$ turns out to be imaginary, and consequently, Eq. (30) becomes an oscillatory function of $R$ that describes the propagation of Cherenkov radiation. In any other case, Eq. (30) decays exponentially with $R$ [even for real dielectric functions, unlike what happens with $\left.G_{j}(r)\right]$ and this is reflected in an exponential dependence of the loss probability with impact parameter, first reported in the nonretarded limit for planar interfaces. ${ }^{10}$

For an electron moving parallel to the $z$ axis with impact parameter $\mathbf{R}_{0}$ inside medium $j_{0}$, the external charge density reads $-2 \pi \delta\left(\mathbf{R}-\mathbf{R}_{0}\right) \delta(\omega-q v)$ and the boundary sources $\phi_{j_{0}}^{e}, \mathbf{A}_{j_{0}}^{e}, \vec{\alpha}$, and $D^{e}$ are proportional to $\delta(\omega-q v)$, so that only the Fourier component $q=\omega / v$ makes a contribution. More precisely, using Eq. (24) with $\mathbf{b}^{\perp}=\mathbf{R}_{0}$ and $\mathbf{b}^{\|}=0$, one has

$$
\phi_{j_{0}}^{e}(\theta, q)=\frac{-4 \pi}{\epsilon_{j_{0}}} K_{0}\left[\frac{\omega\left|\mathbf{R}_{s}(\theta)-\mathbf{R}_{0}\right|}{v \gamma_{j_{0}}}\right] \delta(\omega-q v),
$$

and $\mathbf{A}_{j}^{e}, \vec{\alpha}$, and $D^{e}$ are readily obtained using Eqs. (25), (15), and (18). Therefore, making use of Eq. (29), the linearity of Maxwell's equations leads to

$$
\sigma_{j_{0}}(\theta, z)=\tilde{\sigma}_{j_{0}}\left(\theta, \frac{\omega}{v}\right) \frac{e^{i \omega z / v}}{v}
$$

and

$$
\mathbf{h}_{j_{0}}(\theta, z)=\widetilde{\mathbf{h}}_{j_{0}}\left(\theta, \frac{\omega}{v}\right) \frac{e^{i \omega z / v}}{v},
$$

where the delta function $\delta(\omega-q v)$ has been used and the functions $\widetilde{\sigma}_{j_{0}}$ and $\widetilde{\mathbf{h}}_{j_{0}}$ have a smooth dependence on $\omega$.

Using Eqs. (32) and (33), the integrand on the right hand side of Eq. (28) turns out to be independent of $t$, so that, after dividing by the path length, the electron energy loss probability per unit of path length reduces to 


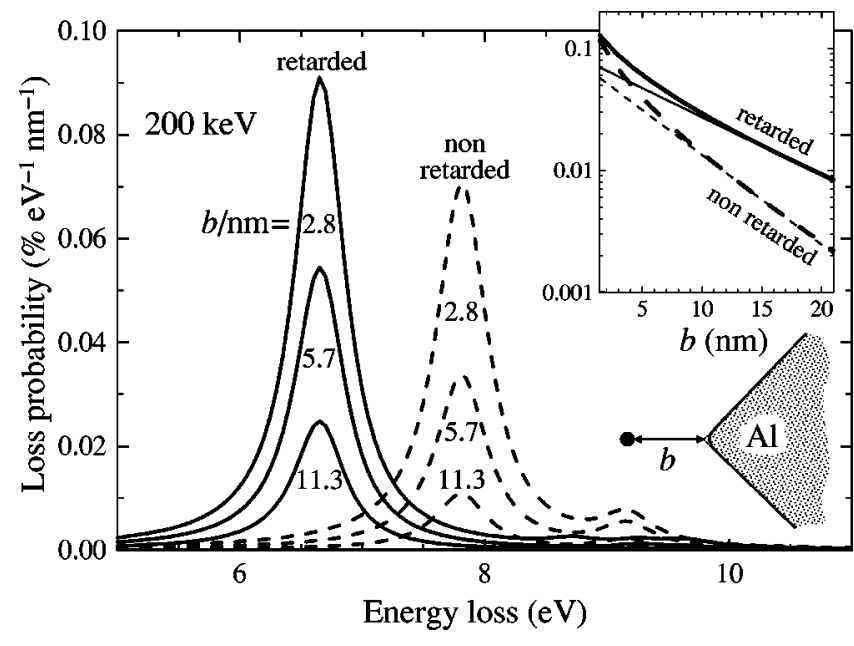

FIG. 3. Impact parameter dependence of the energy loss probability per energy range and path length for $200-\mathrm{keV}$ electrons moving parallel to a hyperbolic $90^{\circ} \mathrm{Al}$ wedge (see inset). Various values of the impact parameter $b$ have been considered, as shown by labels. Retarded (nonretarded) results are represented by solid (broken) curves. The inset shows the height of the corner peak (at $\omega$ $=6.7 \mathrm{eV}$ and $\omega=7.8 \mathrm{eV}$ with and without retardation, respectively) as a function of $b$ (thick curves), compared to pure exponential decay $\sim \exp (-3 \omega b / v \gamma)$ and $\sim \exp (-3 \omega b / v)$ in the retarded and nonretarded cases, respectively (thin lines).

$$
\begin{aligned}
P^{\text {boundary }}(\omega)= & \frac{2}{\pi v^{2}} \int d \theta J(\theta) \operatorname{Im}\left\{K_{0}\left[\frac{\omega\left|\mathbf{R}_{s}(\theta)-\mathbf{R}_{0}\right|}{v \gamma_{j_{0}}}\right]\right. \\
& \left.\times\left[\tilde{\sigma}_{j_{0}}(\theta, \omega / v)-\left(\frac{\mathbf{v}}{c}\right) \cdot \widetilde{\mathbf{h}}_{j_{0}}(\theta, \omega / v)\right]\right\}
\end{aligned}
$$

where $\widetilde{\sigma}_{j_{0}}$ and $\widetilde{\mathbf{h}}_{j_{0}}$ are obtained from Eqs. (20) and (22) by using Eq. (30) for the Green functions and the transformation rule (31) for the interface integrals. The fact that $\nabla_{\mathbf{r}}$ becomes $\left(\nabla_{\mathbf{R}}, i q\right)$ in Fourier space has been used in deriving this equation [see Eq. (29)].

For arbitrarily oriented trajectories, different $q$ components contribute simultaneously, and they have to be summed up to yield the loss probability. ${ }^{30,32}$ This case will not be considered here for simplicity.

Equation (34) has been employed here to calculate the energy loss probability per energy range and path length for electrons moving near different structures made of $\mathrm{Al}, \mathrm{Bi}$, $\mathrm{Al}_{2} \mathrm{O}_{3}, \mathrm{Si}$, and $\mathrm{SiO}_{2}$, and the results have been represented in Figs. 2-6. The response of $\mathrm{Al}$ has been approximated by a Drude dielectric function

$$
\epsilon(\omega)=1-\frac{\omega_{p}^{2}}{\omega(\omega+i \eta)}
$$

with bulk plasma energy $\omega_{p}=15 \mathrm{eV}$ and damping $\eta$ $=0.5 \mathrm{eV}$. The rest of the dielectric functions have been taken from experimental optical data (Ref. 74 for Bi and Ref. 75 for the rest). $\mu_{j}=1$ has been assumed, since these are nonmagnetic materials. For isolated cylinders and wedges, convergence has been achieved with $N=100$ points (see Sec. II B). For coupled cylinders, 100 points per cylinder are required.

Figure 2 shows loss spectra for electrons moving parallel to an aluminum wedge with different velocities and impact parameters, as shown in the insets. The wedge has a sharp hyperbolic profile, with a rounded corner that facilitates convergence with the number of discretization points (see insets in the figure). These spectra share a prominent peak at around $6.5-8 \mathrm{eV}$ coming from the excitation of modes localized in the corner of the wedge (corner peak), as reported in previous studies. ${ }^{30-32}$ When one considers trajectories passing closer to one of the faces of the wedge than to its corner, planar surface modes show up at higher energies, while the strength of the corner loss is diminished [see Figs. 2 (c) and 2(d)]. The effects of retardation in the loss probability (solid curves as compared to the nonretarded dashed curves) are translated into a redshift of the spectral features. The energy shift of the corner peak amounts to $0.6 \mathrm{eV}$ at 100-keV electrons [Figs. 2(a) and 2(c)] and $1.2 \mathrm{eV}$ at 200keV [Figs. 2(b) and 2(d)]. Energy shifts are more noticeable for low-frequency symmetric modes (the corner peak) than for higher-frequency features. The latter are mainly related to both antisymmetric modes ${ }^{30,32}$ and planar modes. This different behavior of the energy shift can be interpreted in terms of retardation effects in the interaction among different regions of the wedge surface: the only source of sign cancellation in the interaction between distant surface regions in the symmetric corner mode, which does not have any node in the plane of the insets of the figure, ${ }^{30,32}$ comes exclusively from the modulation introduced by the factor $\exp ($ iqz), shared by all modes with parallel momentum $q=\omega / v$; however, the induced charges and currents in antisymmetric modes have more nodes than those in symmetric modes, ${ }^{30,32}$ so that sign cancellations in the interaction between distant regions are more effective in the former. Since retardation effects are smaller in the interaction between near regions, and as we have just stated, the near region has a comparatively larger weight within the contribution coming from antisymmetric modes, the latter are less sensitive to the noted energy shifts as compared with the symmetric corner mode.

Following a similar argument, the characteristic mode wavelength along the direction of translational symmetry, $v / \omega$, increases with velocity and this explains why the retardation shift is greater at $200 \mathrm{keV}$ than at $100 \mathrm{keV}$.

The height of the corner peak maximum drops off nearly exponentially with increasing impact parameter, as shown in Figs. 3. For the same velocity, the nonretarded theory predicts a faster decay with impact parameter as compared with the retarded one. This is consistent with the relativistic contraction of the effective impact parameter [see Eq. (30)] observed in planar $^{36}$ and spherical $^{37,38}$ geometries, and also with experiment. ${ }^{76}$ In order to make this more quantitative, the inset of Fig. 3 shows the height of the corner peak as a function of impact parameter $b$ for $200-\mathrm{keV}$ electrons $(v$ $=0.7 c$ ) calculated both with and without inclusion of retardation effects (thick solid and thick broken curves, corresponding to a $b$-independent energy of $\omega_{1} \approx 6.7 \mathrm{eV}$ and $\omega_{2} \approx 7.8 \mathrm{eV}$, respectively). Beyond $10 \mathrm{~nm}$, these curves be- 


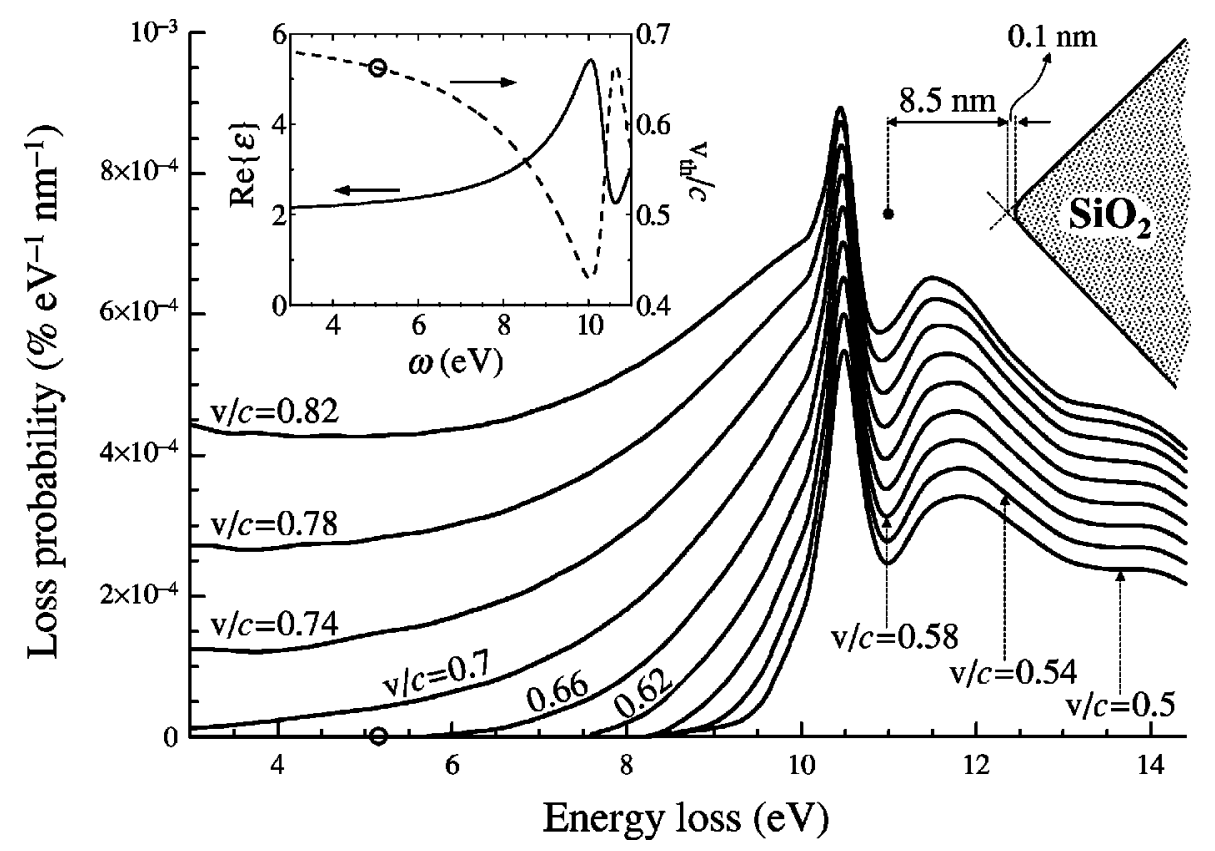

FIG. 4. Energy loss probability per energy range and path length for an electron moving parallel to a hyperbolic $90^{\circ} \mathrm{SiO}_{2}$ wedge. $\mathrm{A}$ section of the wedge is shown in the schematic inset, with the trajectory indicated by a dot. Different electron velocities $v$ have been considered, as shown by labels. The real part of the $\mathrm{SiO}_{2}$ dielectric function, $\operatorname{Re}\{\epsilon\}$, and the threshold velocity $v_{\text {th }}$ above which the Cherenkov condition $v_{\text {th }}>c / \sqrt{\operatorname{Re}\{\epsilon\}}$ is fulfilled have been represented in an inset as a function of energy loss $\omega$.

have similar to the exponentials $\sim \exp \left(-3 \omega_{1} b / v \gamma\right)$ and $\sim \exp \left(-3 \omega_{2} b / v\right)$ (thin curves), respectively, where $\omega_{1} / \gamma$ $=4.8 \mathrm{eV}$ represents the corner mode frequency as observed from the electron frame in the retarded case; both retardation effects via the red shift of the corner peak and relativistic effects via the time expansion in the electron frame (i.e., the $\gamma$ factor) contribute to make slower the decay of the loss probability with impact parameter.

For a $\mathrm{SiO}_{2}$ wedge, the loss probability at low velocities is negligible below $8.5 \mathrm{eV}$, as shown in Fig. 4. The dielectric function is essentially real in that frequency region, and therefore, the medium is unable to absorb energy, so that all losses must be converted into radiation. As the electron velocity increases, the Cherenkov condition $(v / c)^{2} \operatorname{Re}\{\epsilon\}>1$ is fulfilled within an increasingly large energy range below 8.5 $\mathrm{eV}$, and as a result, sizeable losses are produced even though the electron is traveling outside the wedge. The inset of Fig. 4 shows $\operatorname{Re}\{\epsilon\}$ and the threshold velocity above which the Cherenkov condition is satisfied, as a function of energy $\omega$. In particular, the open circle in this inset corresponds to $v$ $=0.66 c$ (see also the horizontal axis of the main figure). The peaks at 10.5 and $11.5 \mathrm{eV}$ are arising from maxima in the imaginary part of the $\mathrm{SiO}_{2}$ dielectric function. The former one is related to localized excitons, whereas the latter one shifts slightly when changing the electron velocity, as different parallel momentum components are involved. A general increase in total losses per unit of path length with increasing velocity is observed within the velocity range under consideration $(v / c=0.5-0.82)$. For high energy losses this effect is due to the role played by the impact parameter; for instance, the surface energy loss per unit of path length for an electron moving parallel to a planar surface scales with the impact parameter $z$ and velocity $v$ as $K_{0}(2 \omega z / v) / v^{2}$, which increases with $v$ within the velocity range under consideration for $z=8.5 \mathrm{~nm}$ and $\omega \sim 12 \mathrm{eV}$. The relativistic contraction of the excitation frequency as seen from the moving electron frame contributes to this effect as well. However, for small impact parameters this velocity dependence can be reversed.

When the electron penetrates a medium, the bulk losses [Eq. (26)] can make significant contributions. This is the case of bismuth cylinders immersed in alumina, which have been recently the subject of EELS studies by Sander el al. ${ }^{77}$ They prepared their samples by pressure injection of bismuth into porous alumina and observed energy losses of 200-keV electrons moving parallel to the cylinders and near the interface of one of them. The results of their experiment are shown in Fig. 5(a) (symbols). Our calculated result (thick solid curve) agrees reasonably well with the experiment. Notice that the calculated results are expressed in absolute units of percentage per eV of energy loss per nm of electron path. The absolute scale in these computations could have been compared with the experimental result if this had included an indication of the specimen thickness, the scattering aperture used, and the number of counts relative to those in the zero loss channel. The 1.2-nm electron beam used in the experiment ${ }^{77}$ ex- $^{-}$ tends partly into bismuth and partly into alumina, so that the loss spectrum picks up bulk losses from those two materials (thin curves). However, the loss spectrum cannot be understood as a simple linear combination of bulk losses in these two materials. Instead, one has to include the contribution of intrinsic interface terms, as calculated from Eq. (34). This results in the partial suppression of bulk losses in favor of interface losses (the so-called begrenzung effect). Furthermore, the $5.5 \mathrm{eV}$ feature is entirely coming from the excitation of interface modes. 

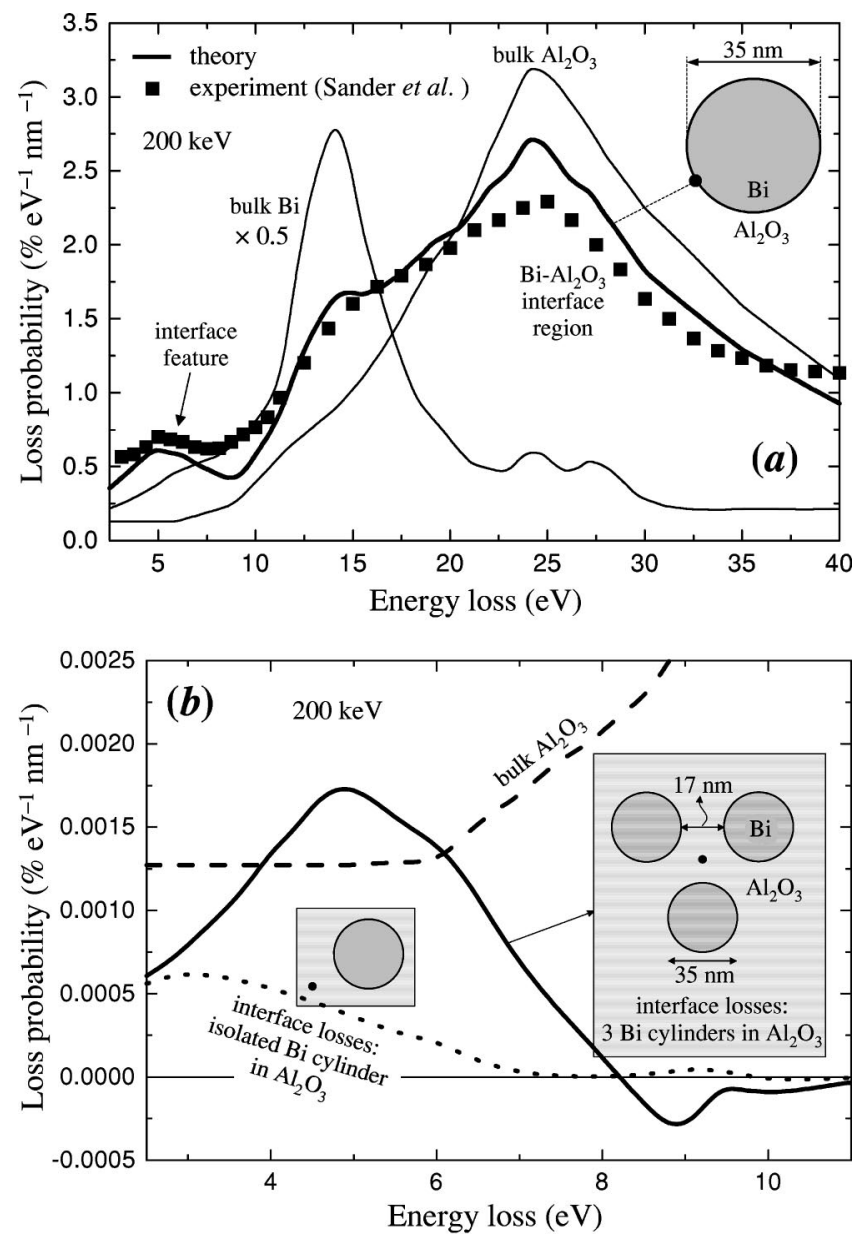

FIG. 5. (a) Energy loss probability for $200-\mathrm{keV}$ electrons passing near the interface of a bismuth cylinder of $35 \mathrm{~nm}$ in diameter immersed in alumina, calculated with the present theory (thick curve) and compared with experimental results by Sander et al. (symbols; Ref. 77). The beam runs parallel to the cylinder and its diameter is $1.2 \mathrm{~nm}$. The bulk loss functions of bismuth and alumina [Eq. (26)] are represented by thin curves for comparison (the probability for bismuth has been multiplied by a factor of 0.5 to improve readability). (b) Boundary contribution to the loss probability [Eq. (34)] for electrons moving in alumina and passing near three bismuth cylinders (solid curve) and near one cylinder (dotted curve), as shown in the insets. The distance from the trajectory to the cylinder centers is $30 \mathrm{~nm}$ in all cases and the separation between cylinder surfaces is $17 \mathrm{~nm}$. The bulk loss function of alumina is represented by a dashed curve [ $P^{\text {bulk }}$, Eq. (26)].

The actual samples consist of triangular lattices of bismuth cylinders. ${ }^{77}$ The relatively large distance of $17 \mathrm{~nm}$ between cylindrical interfaces suppresses to a large extent the contribution of cylinder-cylinder interaction to the energy loss probability, except for low-energy losses and electron trajectories passing far from the interfaces, as shown in Fig. 5(b). The interface contribution to the losses [Eq. (34)] changes quite dramatically when one considers either just one cylinder (dotted curve) or three interacting cylinders (solid curves). This effect should be observable when adding the bulk losses (dashed curve), which are mainly coming from Cherenkov radiation in alumina [the condition $(v / c)^{2}$

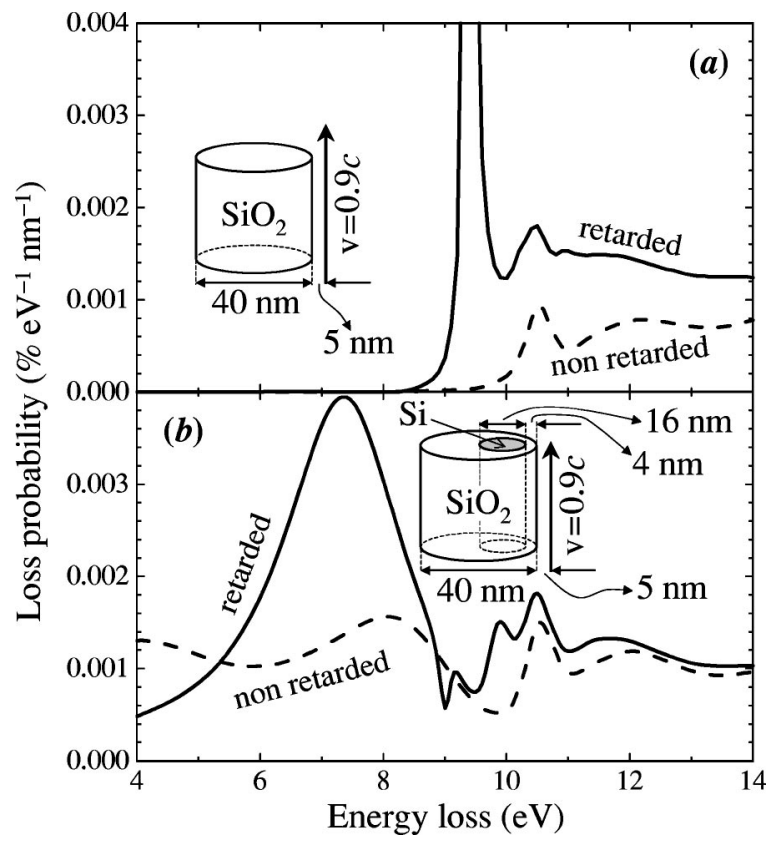

FIG. 6. (a) Energy loss probability per energy range and path length for electrons moving parallel to an infinite $\mathrm{SiO}_{2}$ cylinder of $40 \mathrm{~nm}$ in diameter, as shown in the inset. (b) Same as (a), when the cylinder contains a smaller off-centered Si cylinder of $16 \mathrm{~nm}$. The electron is moving outside the target with velocity $v=0.9 c$ at a distance of $5 \mathrm{~nm}$ from its surface. The results of retarded (nonretarded) calculations have been represented by solid (broken) curves.

$\approx 0.48>1 / \operatorname{Re}\{\epsilon\} \approx 0.31$ is fulfilled within the energy range under consideration]. The dominant effects of the interaction between cylinders in this case is thus connected to Cherenkov radiation that bounces back and forth between cylinders and also to the long spatial range $\sim v \gamma / \omega \approx 35 \mathrm{~nm}$ of the external perturbation for an energy loss of $\omega=5.5 \mathrm{eV}$. For larger energy losses, absorption in alumina is dominant and the interface contribution is very small.

Notice that the interface contribution for three cylinders in Fig. 5(b)(solid curve) takes negative values around $9 \mathrm{eV}$, and this is again a manifestation of the begrenzung effect as a correction to the bulk loss. The same experimental work ${ }^{77}$ reports on the observation of slight bulk-plasmon shifting and broadening in isolated bismuth cylinders with diameters in the range of $30-90 \mathrm{~nm}$, which the authors attribute to quantum confinement effects in the response. The present theory does not reproduce this finding, and this can be due to quantum confinement related to dispersion in the bulk response of bismuth, which is not incorporated here.

Figure 6(b) illustrates a structure composed of two different materials for an electron running parallel to a $\mathrm{SiO}_{2}$ cylinder that contains a smaller $\mathrm{Si}$ cylinder inside. The results are compared with a homogeneous $\mathrm{SiO}_{2}$ cylinder with the same dimensions in Fig. 6(a). Retardation effects and the presence of the Si core dominate the low-energy part of the spectra.

A sharp peak, corresponding to a transverse electromagnetic wave within the homogeneous cylinder, is observed at around $9.4 \mathrm{eV}$ in Fig. 6(a) (solid curve). This peak, associated to $m= \pm 1$ modes of the cylinder, is completely missed 
by nonretarded calculations (thin broken curve). Our simulations show that this feature shifts towards lower energies with increasing electron velocity $(7.3 \mathrm{eV}$ for $v / c=0.999$ and $6.6 \mathrm{eV}$ for $v / c=0.9999)$, and this is well reproduced by the analytical dispersion relation of the $m=1$ mode for the isolated cylinder. ${ }^{78}$ Furthermore, the width of this peak is proportional to the imaginary part of the dielectric function $\epsilon_{i}$, which is negligibly small in the $\mathrm{SiO}_{2}$ gap region, although the peak height is inversely proportional to $\epsilon_{i}$, so that the total peak area remains approximately independent of $\epsilon_{i}$. This peculiar feature could be used to determine chargedparticle velocities via the wavelength of the radiation that would be emitted when the cylinder is abruptly terminated.

Despite the fact that the bulk Cherenkov condition is fulfilled within the whole energy range of Fig. 6(a), no significant losses are observed below $8.5 \mathrm{eV}$, unlike what happens in the silica wedge of Fig. 4. However, in a medium that is restricted laterally with respect to the electron trajectory, the Cherenkov radiation can only exist for specific quantized modes of the structure, as in the guided mode observed at 9.4 $\mathrm{eV}$ in the cylinder.

The losses are changed substantially by the introduction of an off-centered core of Si [see Fig. 6(b)]. The guided Cherenkov peak at $9.4 \mathrm{eV}$ largely disappears and new losses emerge in the 6-8 eV region, where the $\mathrm{Si}$ has metallic behavior (the dielectric function has a negative real part and a significant imaginary part). This loss spectrum, however, still depends profoundly on the existence of the surrounding $\mathrm{SiO}_{2}$ region.

\section{EELS IN AXIALLY SYMMETRIC INTERFACES}

When the sample is invariant under rotations around a particular direction (e.g., the $z$ axis), its interfaces can be parametrized as $\mathbf{s}(\theta, \phi)=\left[\rho_{s}(\theta) \cos \phi, \rho_{s}(\theta) \sin \phi, z_{s}(\theta)\right]$, where $\rho_{s}$ is the distance to the $z$ axis, $\phi$ is the azimuthal angle, and $\theta$ is a parameter. Then, the different magnitudes defined in Sec. II can be expressed in terms of a Fourier series such as

$$
\sigma_{j}(\theta, \phi)=\frac{1}{2 \pi} \sum_{m} \sigma_{j}^{m}(\theta) e^{i m \phi},
$$

where

$$
\sigma_{j}^{m}(\theta)=\int_{-\pi}^{\pi} d \phi \sigma_{j}(\theta, \phi) e^{-i m \phi} .
$$

In particular, the Fourier components of the Green functions are

$$
G_{j}^{m}\left(\theta, \theta^{\prime}\right)=\int_{-\pi}^{\pi} d \phi \frac{e^{i k_{j} d\left(\theta, \theta^{\prime}, \phi\right)}}{d\left(\theta, \theta^{\prime}, \phi\right)} e^{-i m \phi},
$$

where

$$
\begin{aligned}
d\left(\theta, \theta^{\prime}, \phi\right)= & \left\{\rho_{s}(\theta)^{2}+\rho_{s}\left(\theta^{\prime}\right)^{2}-2 \rho_{s}(\theta) \rho_{s}\left(\theta^{\prime}\right) \cos \phi\right. \\
& \left.+\left[z_{s}(\theta)-z_{s}\left(\theta^{\prime}\right)\right]^{2}\right\}^{1 / 2} .
\end{aligned}
$$

Moreover, the surface integrals can be performed making the substitution

$$
\int_{S} \rightarrow \int d \theta \rho_{S}(\theta) M(\theta)
$$

where $M(\theta)=\sqrt{\rho_{s}^{\prime}(\theta)^{2}+z_{s}^{\prime}(\theta)^{2}}$ and the integral over $\phi$ reduces to a multiplication for each component $m$ in virtue of the convolution theorem (i.e., each $m$ component is decoupled from the rest).

For an electron whose trajectory is fully contained inside medium $j_{0}$ and is defined by $x=b, y=0$, and $z=v t$, the external boundary source (24) can be written ${ }^{38}$

$$
\phi_{j_{0}}^{\mathrm{e}, m}(\theta)=\frac{-4 \pi}{v \epsilon_{j_{0}}} e^{i \omega z_{s}(\theta) / v} I_{m}\left(\frac{\omega R_{<}}{v \gamma_{j_{0}}}\right) K_{m}\left(\frac{\omega R_{>}}{v \gamma_{j_{0}}}\right),
$$

where $\quad R_{<}=\min \left\{\rho_{s}(\theta), b\right\}, \quad R_{>}=\max \left\{\rho_{s}(\theta), b\right\}, \quad$ and $\quad \gamma_{j_{0}}$ $=1 / \sqrt{1-\epsilon_{j_{0}} \mu_{j_{0}} v^{2} / c^{2}}$. Equations (25), (15), and (18) can be used to obtain the rest of the equivalent boundary sources.

It is convenient to project vector quantities such as $\mathbf{h}_{j}$ onto the cylindrical frame defined by the vectors $\hat{\rho}, \hat{\phi}$, and $\hat{z}$. The identities needed to obtain the Fourier components of such projections are given in Appendix B. Solving the projected self-consistent system of equations for the boundary charges, one obtains $\sigma_{j_{0}}^{m}$ and $\mathbf{h}_{j_{0}}^{m}$, in terms of which the loss probability [Eq. (28)] reduces to

$$
\begin{aligned}
\Gamma^{\text {boundary }}(\omega)= & \frac{2}{\pi v} \sum_{m=-\infty}^{\infty} \int d \theta \rho_{s}(\theta) M(\theta) \\
& \times \operatorname{Im}\left\{I_{m}\left(\frac{\omega R_{<}}{v \gamma_{j_{0}}}\right) K_{m}\left(\frac{\omega R_{>}}{v \gamma_{j_{0}}}\right)\right. \\
& \left.\times e^{-i \omega z_{s}(\theta) / v}\left[\sigma_{j_{0}}^{m}(\theta)-\left(\frac{\mathbf{v}}{c}\right) \cdot \mathbf{h}_{j_{0}}^{m}(\theta)\right]\right\} .
\end{aligned}
$$

Some examples of application of Eq. (38) have been considered in Figs. 7-11. In this case, the damping of the dielectric function for $\mathrm{Al}$ has been taken $\eta=1.06 \mathrm{eV}$.

Figure 7 shows the loss probability for electrons passing near two $\mathrm{Al}$ spheres along a direction parallel to the bond vector. Different distances between the surfaces of the two spheres have been considered as shown by labels. Notice the huge effect of retardation both for isolated spheres and for spheres separated by $1 \mathrm{~nm}$ : the broken curves stand for the nonretarded result and retardation (solid curves) leads to various Mie resonances and also to an enhancement of the effect of the interaction between the spheres at low energies. In fact, the sphere radius $a=50 \mathrm{~nm}$ is large enough to produce a difference in phase of $\omega a / c=116^{\circ}$ when the electromagnetic signal travels along the length of the radius with a representative energy of $\omega=8 \mathrm{eV}$. The interaction between the spheres is small down to a separation of $5 \mathrm{~nm}$ (i.e., a tenth of the radius in this case), below which a low energy peak emerges at $\approx 2 \mathrm{eV}$. However, the nonretarded results are dominated by the region of the spheres close to the elec- 


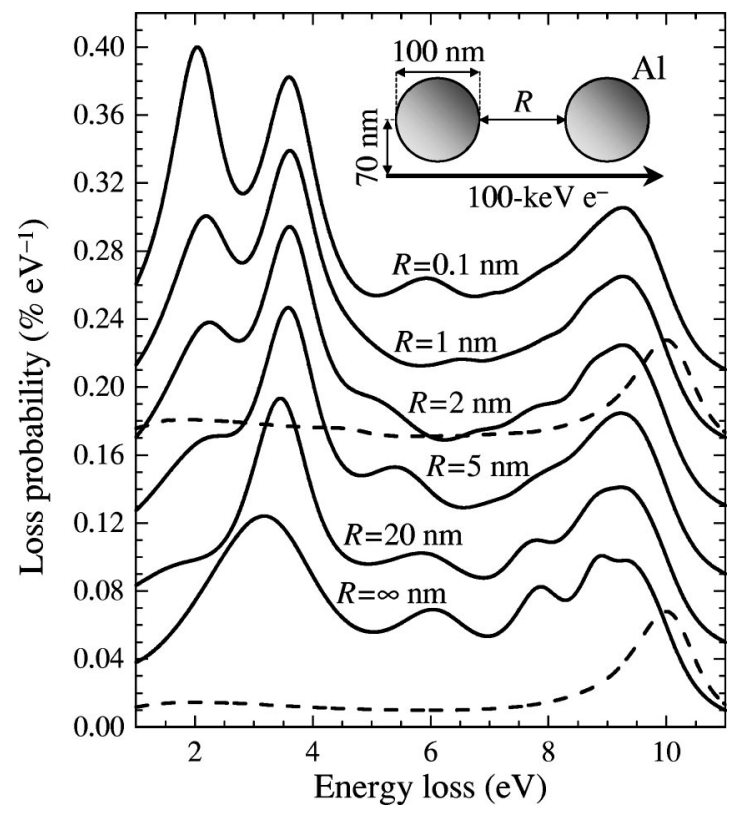

FIG. 7. Energy loss probability per energy range for $100-\mathrm{keV}$ electrons passing at a distance $b=70 \mathrm{~nm}$ from the centers of two $\mathrm{Al}$ spheres of $100 \mathrm{~nm}$ in diameter. Different values of the distance $R$ between sphere surfaces have been considered, as shown by labels. The nonretarded results have been plotted for comparison for $R$ $=\infty$ and for $R=1 \mathrm{~nm}$ (broken curves). Consecutive curves have been shifted $0.04 \% \mathrm{eV}^{-1}$ upwards to improve readability.

tron trajectory, so that the mutual nonretarded interaction between the spheres plays a minor role for this particular geometry; from all the nonretarded multipole modes of the sphere, $\omega_{p} \sqrt{l /(2 l+1)}$, high values of $l$ are dominant, leading to a peak in the loss probability near the planar surface energy $\omega_{p} / \sqrt{2}=10.6 \mathrm{eV}$ (actually, the peak shows up at $\approx 10 \mathrm{eV}$ both for isolated spheres and for spheres separated by $1 \mathrm{~nm}$ ): the electron sees the sphere surfaces as nearly planar in the nonretarded approximation.

The loss probability experienced by an electron passing near an aluminum torus has been considered in Fig. 8, In particular, Fig. 8(a) represents the contribution of $m$ modes summed up to a given maximum value. A relatively large number of $m$ 's $(\approx 12)$ is necessary to reach convergence in this case of a grazing trajectory. The contribution of separate $m$ 's is represented in Fig. 8(b). Nonretarded results (broken curves) are in good agreement with retarded calculations (solid curves) for high-order modes $(|m|>4)$, since these involve induced charges and currents characterized by a large number of nodes. As discussed for the wedge above, the interaction between regions of the sample that are relatively far apart then averages out, and hence, retardation is not relevant in the remaining short-range interaction.

When several tori are brought together [Fig. 9(a)], the interaction among them produces splitting and mixing of low-energy spectral features. The position of the prominent peak at around $9.6 \mathrm{eV}$ is quite insensitive to both retardation effects and tori coupling. Retardation effects (solid curves) show up as a shift of the low-energy features of the spectra towards lower energies with respect to nonretarded results (broken curves).

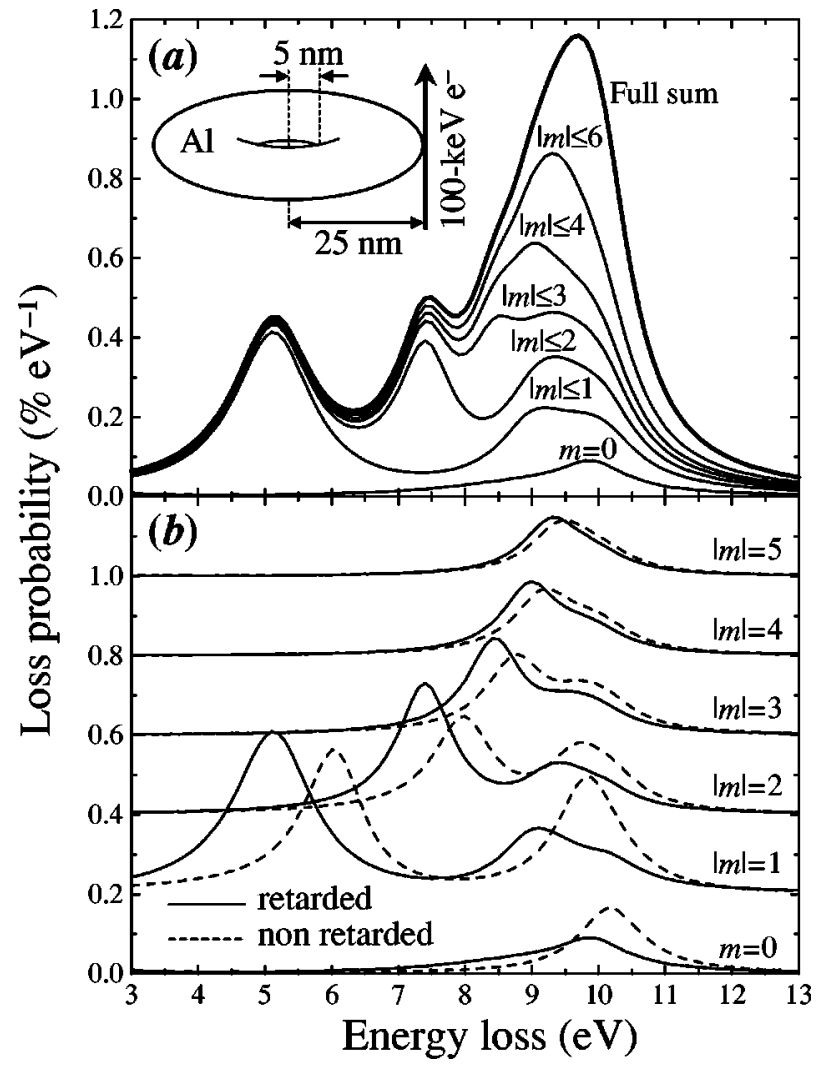

FIG. 8. (a) Energy loss probability per energy range for $100-\mathrm{keV}$ electrons moving parallel to the axis of symmetry of an aluminum torus. The inner (outer) radius of the torus is $5 \mathrm{~nm}(25 \mathrm{~nm})$ and its height is $20 \mathrm{~nm}$. The trajectory is grazing with respect to the torus surface, as shown in the inset. The contributions of different $m$ azimuthal modes up to a maximum finite value (see labels) are compared with the fully converged sum for all $m$ 's. (b) Under the same conditions as in (a), separate contribution of different $m$ 's calculated with and without the inclusion of retardation effects (solid and broken curves, respectively). Consecutive curves have been shifted $0.2 \% \mathrm{eV}^{-1}$ upwards to improve readability.

For an electron trajectory coinciding with the axis of symmetry of the tori, only $m=0$ contributes to the loss probability [Figs. 9(b) and 9(c)]. Nonretarded results (broken curves) are in relatively good agreement with retarded results (solid curves) in Fig. 9(b). This is not the case in Fig. 9(c), where the larger size of the target enhances the effects of retardation in the electromagnetic signal coupling different parts of the surfaces.

Incidentally, modes of energy above the classical surface plasmon energy $\omega_{p} / \sqrt{2}$ are efficiently excited in the geometry of Figs. 9(b) and 9(c). This is connected to the fact that the electron is interacting mainly with surface regions of negative curvature (the hole of the tori), ${ }^{30}$ unlike what happens in Fig. 9(a).

Figure 10 shows EELS spectra calculated for hemispheres of Al of radius $a=10 \mathrm{~nm}$ in (a) and $a=20 \mathrm{~nm}$ in (b). The position of the feature at around $6.9 \mathrm{eV}$ resulting from nonretarded calculations (broken curves) does not change from (a) to (b). This reflects the fact that the mode frequencies do not change under scaling transformations when retardation is 


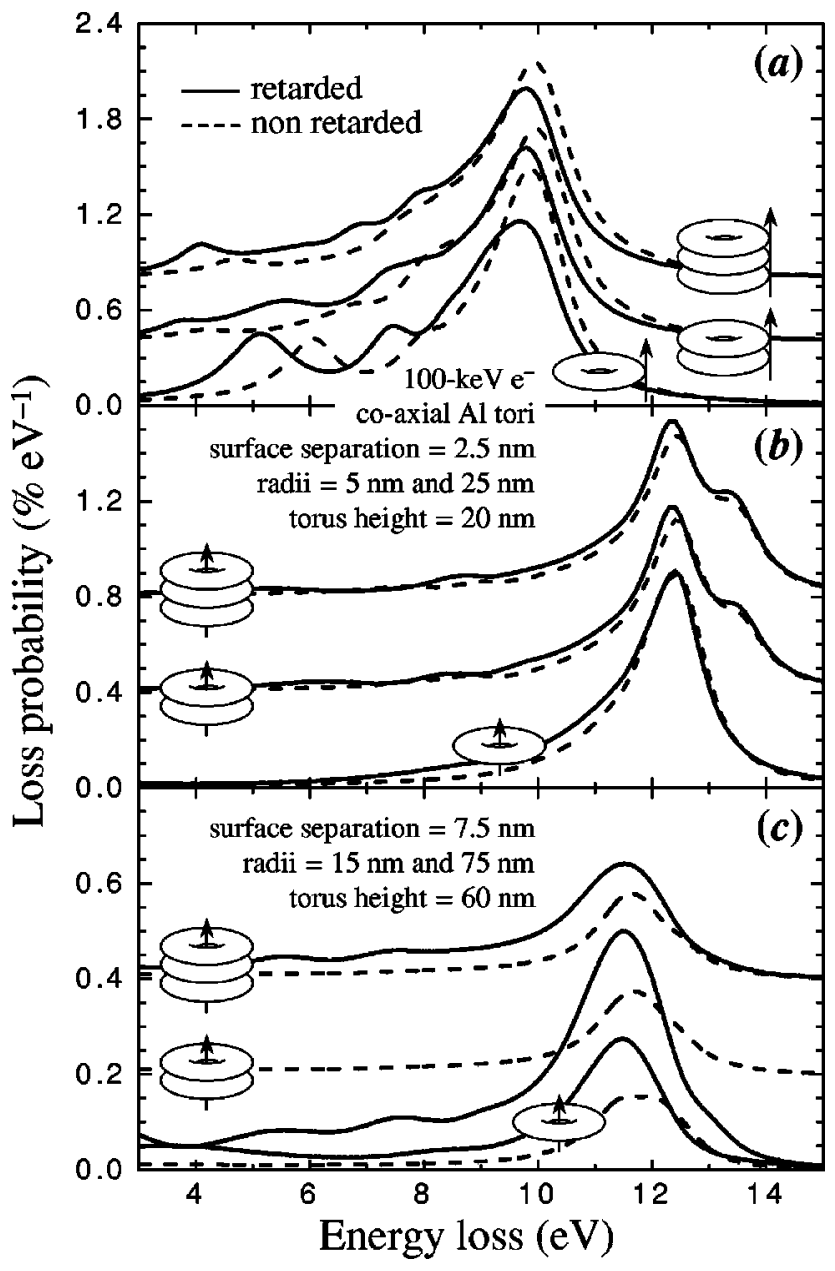

FIG. 9. (a) Energy loss probability per energy range for a 100$\mathrm{keV}$ electron moving parallel to the axis of symmetry of $1-3$ coaxial Al tori. The inner (outer) radius of the tori is $5 \mathrm{~nm}(25 \mathrm{~nm})$ and their height is $20 \mathrm{~nm}$. The separation between consecutive tori surfaces is $2.5 \mathrm{~nm}$. The results of retarded (nonretarded) calculations have been represented by solid (broken) curves. The trajectory is grazing with respect to the tori, as shown in the insets. The probability has been divided by the number of tori in each case. Consecutive curves have been shifted $0.4 \% \mathrm{eV}^{-1}$ upwards to improve readability. (b) Same as (a) for an electron moving along the axis of symmetry. (c) Same as (b) for tori with an inner (outer) radius of 15 $\mathrm{nm}(75 \mathrm{~nm})$, a height of $60 \mathrm{~nm}$, and a surface separation of $7.5 \mathrm{~nm}$. Consecutive curves have been shifted $0.2 \% \mathrm{eV}^{-1}$ in this case.

ignored. Retardation effects (solid curves) are stronger in (b) due to the larger radius of the hemisphere, and this is once more translated into a shift of the spectral features towards lower energies, which adds to the geometrical red shift undergone by the modes of hemispheres as compared to those of spheres. ${ }^{79,20}$

The features of the energy loss spectra could be potentially employed to investigate the shape of an object in the direction perpendicular to the electron beam. This is illustrated in Fig. 11, where several Al ellipsoids have been considered, all of them having in common the same projection along the direction of the electron trajectory: a circle of radius $10 \mathrm{~nm}$. A strong dependence of the position and magnitude of the different features of the loss spectra on the elon-

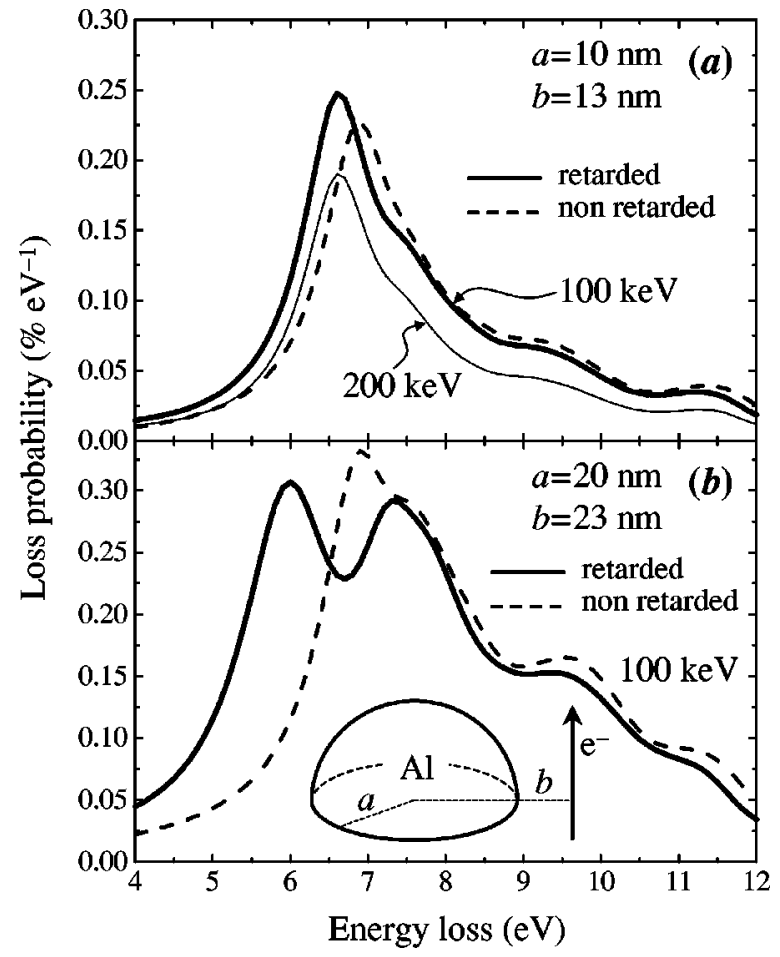

FIG. 10. Energy loss probability per energy range for an electron passing near an aluminum hemisphere of radius $a$. The electron moves parallel to the axis of symmetry of the hemisphere with impact parameter $b$. (a) $a=10 \mathrm{~nm}$ and $b=13 \mathrm{~nm}$. (b) $a=20 \mathrm{~nm}$ and $b=23 \mathrm{~nm}$. The electron energy is $100 \mathrm{keV}$, except in the thin curve in (a), calculated for $200 \mathrm{keV}$. The results of retarded (nonretarded) calculations have been represented by solid (broken) curves.

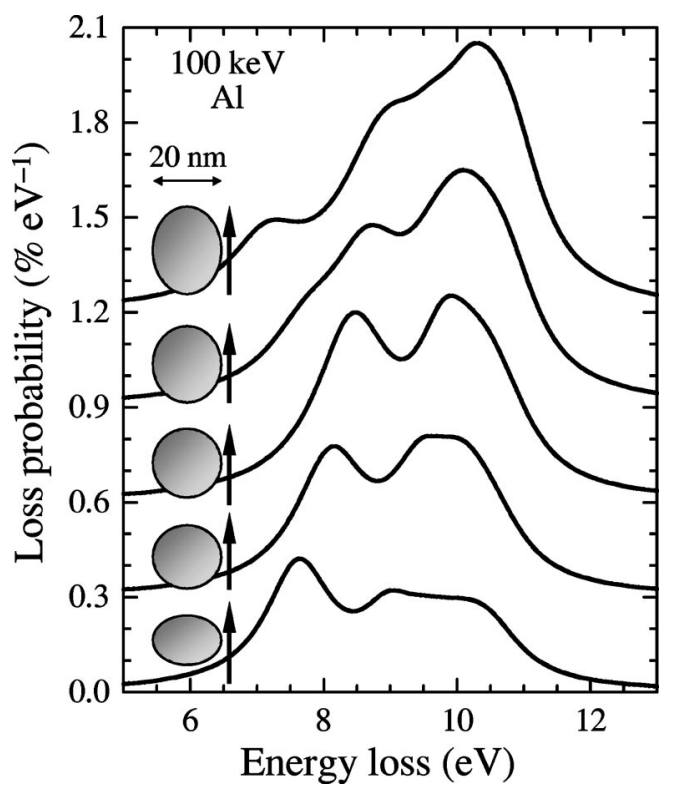

FIG. 11. Energy loss probability per energy range for a 100-keV electron passing at a distance of $1 \mathrm{~nm}$ from the surface of an aluminum cylindrical ellipsoid. The electron moves parallel to the axis of symmetry, along which the semi-axis of the ellipsoid takes different values $(14,12,10,8$, and $6 \mathrm{~nm}$ from top to bottom; see insets). The other semiaxes are $10 \mathrm{~nm}$ in all cases. 
gation of the ellipsoid is observed. The hemisphere of Fig. 10(a) has also the same projection and gives rise to a very different spectrum for the same electron energy.

\section{CONCLUDING REMARKS}

A general technique has been presented to calculate electron energy losses in the presence of arbitrarily shaped dielectrics by solving Maxwell's equations, making use of boundary charges and currents to represent the response of the system. This permits us to reduce the three-dimensional electromagnetic field to two-dimensional surface integral equations that are solved self-consistently by discretizing the integrals. The method allows one to deal with magnetic materials as well as heterostructures involving an arbitrary number of different materials. It is also very suitable to describe metals with thin skin depths and high dielectric contrast.

Detailed consideration has been given to the cases of translationally invariant interfaces and axially symmetric interfaces, where use of symmetry has been made to ease the numerical effort by reducing the problem to effectively one dimension.

The effects of retardation have been proved to be dramatic when the size of the objects under consideration is large, even for electron energies of $100 \mathrm{keV}$ (see Fig. 7). These effects are connected to the variation in the phase of an electromagnetic signal when it travels across the characteristic length $a$ of a given object, so that when $a$ is large, the phase difference $\omega a / c$ becomes also large. A general shift of the excitation modes towards lower energies has been observed when retardation is incorporated, as compared to a nonretarded description obtained from Poisson's equation. This behavior can be qualitatively understood by considering an object described by a frequency-independent dielectric function; then, one can argue that the frequency $\omega$ of a given eigenmode will scale with $a$ in such a way that $\omega a / c$ is a dimensionless constant number, and hence, the larger the radius $a$, the smaller the frequency $\omega$.

The effect of the electron velocity consists in changing the weight attributed to each of the excitation modes in the loss spectra. For finite objects, where only a small number of modes contribute efficiently to the energy loss, varying the velocity does not produce any significant energy shift in the features of the spectra. However, for unbound systems, similar to the wedges considered in Sec. IV, the number of relevant modes is infinite (they are labeled by the momentum $q$ in the case of translationally invariant objects such as those discussed in Sec. IV), and a different coupling between target and electron due to different velocities results in energy shifts of the features of the loss spectra with varying velocity (see Fig. 2).

Radiative energy losses are predicted when the Cherenkov condition $(v / c)^{2} \operatorname{Re}\{\epsilon\}>1$ is satisfied even for electrons moving outside a material that is not corrugated along the direction of motion (see Fig. 4). This effect is observed in the case of targets unbound in at least one direction perpendicular to the electron velocity (e.g., the wedge of Fig. 4), but not in the homogeneous silica cylinder discussed in Fig. 6.

Low-order modes are more sensitive to retardation than high-order modes, since they involve strong interaction between regions of the interfaces that are relatively far apart. However, they require more extended external perturbations to be excited, and larger electron velocities facilitate this (the spatial range of the external field set up by the electron scales as $\approx v / \omega$ with the velocity).

Energy shift and splitting of spectral features is also observed due to the interaction between neighboring objects, as it has been shown for the cases of cylindrical holes, spheres, and tori.

Finally, iteration techniques such as the recursion method $^{80,81}$ have proved to be useful in solving Poisson's equation, ${ }^{82,83}$ and a similar approach could be applied within the present formalism. Further research in this direction is in progress.

\section{ACKNOWLEDGMENTS}

One of the authors (F.J.G.A.) wants to acknowledge help and support from the Spanish Ministerio de Ciencia y Tecnología and the Basque Government.

\section{APPENDIX A: THE GRADIENT OF THE GREEN FUNCTIONS AT THE INTERFACE}

This appendix is devoted to derive expressions for the normal derivatives of the Green function (8) at the interface, where the divergence of $G_{j}\left(\left|\mathbf{s}-\mathbf{s}^{\prime}\right|\right)$ when $\mathbf{s}=\mathbf{s}^{\prime}$ will be shown to contribute with a surface $\delta$ function. The gradient of the Green function (8) taken from both sides of the interface can be written

$$
\lim _{\eta \rightarrow 0^{+}} \nabla_{\mathbf{s}} G_{j}\left(\left|\mathbf{s} \pm \eta \mathbf{n}_{s}-\mathbf{s}^{\prime}\right|\right)=\nabla_{\mathbf{s}} G_{j}(|\mathbf{s}-\mathbf{s}|)+\mathbf{n}_{s} T^{ \pm}\left(\mathbf{s}, \mathbf{s}^{\prime}\right),
$$

where

$$
T^{ \pm}\left(\mathbf{s}, \mathbf{s}^{\prime}\right)= \pm \lim _{\eta \rightarrow 0^{+}} \frac{\eta}{\left|\mathbf{s} \pm \eta \mathbf{n}_{s}-\mathbf{s}^{\prime}\right|} G_{j}^{\prime}\left(\left|\mathbf{s} \pm \eta \mathbf{n}_{s}-\mathbf{s}^{\prime}\right|\right)
$$

and $G_{j}^{\prime}(r)$ is the derivative of $G_{j}(r)$ with respect to $r$. Multiplying $T^{ \pm}$by an arbitrary function $f\left(\mathbf{s}^{\prime}\right)$, integrating over $\mathbf{s}^{\prime}$, and changing the variable of integration to $\mathbf{w}=(\mathbf{s}$ $\left.-\mathbf{s}^{\prime}\right) / \eta$, one finds

$$
\begin{aligned}
& \int_{S} d \mathbf{s}^{\prime} T^{ \pm}\left(\mathbf{s}, \mathbf{s}^{\prime}\right) f\left(\mathbf{s}^{\prime}\right) \\
& = \pm \lim _{\eta \rightarrow 0^{+}} \int d \mathbf{w} \frac{\eta^{3}}{\left|\mathbf{s} \pm \eta \mathbf{n}_{s}-\mathbf{s}^{\prime}\right|} G_{j}^{\prime}\left(\left|\mathbf{s} \pm \eta \mathbf{n}_{s}-\mathbf{s}^{\prime}\right|\right) \\
& \times f(\mathbf{s}-\eta \mathbf{w}),
\end{aligned}
$$

where the identity $d \mathbf{s}^{\prime}=\eta^{2} d \mathbf{w}$ has been used. The integrand on the right hand side of this expression vanishes in the $\eta$ $\rightarrow 0$ limit except near $\mathbf{s}=\mathbf{s}^{\prime}$. Then, noticing that $G_{j}(r) \approx 1 / r$ when $\left|k_{j} r\right| \ll 1$, one can approximate $G_{j}^{\prime}\left(\left|\mathbf{s} \pm \eta \mathbf{n}_{s}-\mathbf{s}^{\prime}\right|\right)$ by $-1 /\left|\mathbf{s} \pm \eta \mathbf{n}_{s}-\mathbf{s}^{\prime}\right|^{2}$ in Eq. (A1). Moreover, one has $\mid \mathbf{s} \pm \eta \mathbf{n}_{s}$ 
$-\mathbf{s}^{\prime} \mid \approx \eta \sqrt{w^{2}+1}$, provided the interface is smooth, since $\mathbf{w}$ becomes normal to $\mathbf{n}_{s}$ when $\mathbf{s}^{\prime} \rightarrow \mathbf{s}$. Finally, one obtains

$$
\int_{S} d \mathbf{s}^{\prime} T^{ \pm}\left(\mathbf{s}, \mathbf{s}^{\prime}\right) f\left(\mathbf{s}^{\prime}\right)=\mp \int \frac{d \mathbf{w}}{\left(w^{2}+1\right)^{3 / 2}} f(\mathbf{s})=\mp 2 \pi f(\mathbf{s}),
$$

from where the equation

$$
\lim _{\eta \rightarrow 0^{+}} \nabla_{\mathbf{s}} G_{j}\left(\left|\mathbf{s} \pm \eta \mathbf{n}_{s}-\mathbf{s}^{\prime}\right|\right)=\nabla_{\mathbf{s}} G_{j}\left(\left|\mathbf{s}-\mathbf{s}^{\prime}\right|\right) \mp 2 \pi \mathbf{n}_{s} \delta\left(\mathbf{s}-\mathbf{s}^{\prime}\right)
$$

can be derived.

\section{APPENDIX B: \\ USEFUL EXPRESSIONS FOR AXIAL SYMMETRY}

This appendix contains the basic identities that permit one to deal with Fourier transforms of the vector quantities involved in the self-consistent surface-integral equations for the electromagnetic field in the case of axially symmetric interfaces. Vector quantities $\mathbf{h}$ will be projected onto the cylindrical frame defined by the vectors $\hat{\rho}, \hat{\phi}$, and $\hat{z}$. Given any operator $G$, one has

$$
\begin{gathered}
{[\hat{\rho} G \cdot \hat{\rho}]^{m}=[\hat{\phi} G \cdot \hat{\phi}]^{m}=\frac{1}{2}\left(G^{m+1}+G^{m-1}\right),} \\
{[\hat{\rho} G \cdot \hat{\phi}]^{m}=-[\hat{\phi} G \cdot \hat{\rho}]^{m}=\frac{i}{2}\left(G^{m+1}-G^{m-1}\right),}
\end{gathered}
$$

$$
[\hat{z} G \cdot \hat{\phi}]^{m}=[\hat{z} G \cdot \hat{\rho}]^{m}=0
$$

and

$$
[\hat{z} G \cdot \hat{z}]^{m}=G^{m},
$$

where $m$ is the Fourier component index, as defined by Eqs. (35) and (36). Using these expressions, the components of matrix-vector products are readily calculated as

$$
\begin{aligned}
{[G \mathbf{h}]^{m}=} & \hat{\rho}\left(G^{m+1} h_{+}^{m}+G^{m-1} h_{-}^{m}\right) \\
& +i \hat{\phi}\left(-G^{m+1} h_{+}^{m}+G^{m-1} h_{-}^{m}\right)+\hat{z} G^{m}[\hat{z} \cdot \mathbf{h}]^{m},
\end{aligned}
$$

where $h_{ \pm}^{m}=(1 / 2)[(\hat{\rho} \pm i \hat{\phi}) \cdot \mathbf{h}]^{m}$. Also, the identities

$$
[\hat{x} \cdot \mathbf{h}]^{m}=h_{-}^{m+1}+h_{+}^{m-1}
$$

and

$$
[\hat{y} \cdot \mathbf{h}]^{m}=i\left(h_{-}^{m+1}-h_{+}^{m-1}\right)
$$

are useful when projections on the $x$ and $y$ axis are needed. Finally, the inverse of these identities are

$$
[\hat{\rho} \cdot \mathbf{h}]^{m}=\frac{1}{2}\left\{[(\hat{x}-i \hat{y}) \cdot \mathbf{h}]^{m-1}+[(\hat{x}+i \hat{y}) \cdot \mathbf{h}]^{m+1}\right\}
$$

and

$$
[\hat{\phi} \cdot \mathbf{h}]^{m}=\frac{i}{2}\left\{[(\hat{x}-i \hat{y}) \cdot \mathbf{h}]^{m-1}-[(\hat{x}+i \hat{y}) \cdot \mathbf{h}]^{m+1}\right\} .
$$

*Email address: jga@sw.ehu.es

${ }^{1}$ P.D. Nellist and S.J. Pennycook, Phys. Rev. Lett. 81, 4156 (1998).

${ }^{2}$ L.D. Marks, Solid State Commun. 43, 727 (1982).

${ }^{3}$ J.M. Cowley, Phys. Rev. B 25, 1401 (1982).

${ }^{4}$ A. Howie and R.H. Milne, Ultramicroscopy 18, 427 (1985).

${ }^{5}$ R.H. Ritchie and A. Howie, Philos. Mag. A 58, 753 (1988).

${ }^{6}$ R.H. Ritchie, Phys. Rev. 106, 874 (1957).

${ }^{7}$ L. Dobrzynski and A.A. Maradudin, Phys. Rev. B 6, 3810 (1972).

${ }^{8}$ L.C. Davis, Phys. Rev. B 14, 5523 (1976).

${ }^{9}$ A. Eguiluz and A.A. Maradudin, Phys. Rev. B 14, 5526 (1976).

${ }^{10}$ P.M. Echenique and J.B. Pendry, J. Phys. C 8, 2936 (1975).

${ }^{11}$ P.M. Echenique, J. Bausells, and A. Rivacoba, Phys. Rev. B 35, 1521 (1987).

${ }^{12}$ T.L. Ferrell and P.M. Echenique, Phys. Rev. Lett. 55, 1526 (1985).

${ }^{13}$ P.M. Echenique, A. Howie, and D.J. Wheatley, Philos. Mag. B 56, 335 (1987).

${ }^{14}$ J. L. Peng, R. P. Fehlhaber, L. A. Bursill, and D. G. McCulloch, J. Appl. Phys. 89, 6204 (2001).

${ }^{15}$ R. García-Molina, A. Gras-Martí, and R.H. Ritchie, Phys. Rev. B 31, 121 (1985).

${ }^{16}$ B.L. Illman, V.E. Anderson, R.J. Warmack, and T.L. Ferrell, Phys. Rev. B 38, 3045 (1988).

${ }^{17}$ N. Zabala, A. Rivacoba, and P.M. Echenique, Surf. Sci. 209, 465 (1989).

${ }^{18}$ M. Schmeits, Phys. Rev. B 39, 7567 (1989).

${ }^{19}$ A. Rivacoba, N. Zabala, and P.M. Echenique, Phys. Rev. Lett. 69, 3362 (1992).
${ }^{20}$ J. Aizpurua, A. Rivacoba, and S.P. Apell, Phys. Rev. B 54, 2901 (1996).

${ }^{21}$ A. Rivacoba, S.P. Apell, and N. Zabala, Nucl. Instrum. Methods Phys. Res. B 96, 465 (1995).

${ }^{22}$ N. Zabala, A. Rivacoba, and P.M. Echenique, Phys. Rev. B 56, 7623 (1997).

${ }^{23}$ L. Fu, P.B. Macedo, and L. Resca, Phys. Rev. B 47, 13818 (1993).

${ }^{24}$ L. Fu and L. Resca, Phys. Rev. B 47, 16194 (1993).

${ }^{25}$ R.G. Barrera and R. Fuchs, Phys. Rev. B 52, 3256 (1995).

${ }^{26}$ C.I. Mendoza, R.G. Barrera, and R. Fuchs, Phys. Rev. B 57, 11193 (1998).

${ }^{27}$ R. Fuchs, Phys. Rev. B 11, 1732 (1975).

${ }^{28}$ F. Ouyang and M. Isaacson, Philos. Mag. B 60, 481 (1989).

${ }^{29}$ F. Ouyang and M. Isaacson, Ultramicroscopy 31, 345 (1989).

${ }^{30}$ F.J. García de Abajo and J. Aizpurua, Phys. Rev. B 56, 15873 (1997).

${ }^{31}$ J. Aizpurua, B. Rafferty, F.J. García de Abajo, and A. Howie, Inst. Phys. Conf. Ser. 153, 277 (1997).

${ }^{32}$ J. Aizpurua, A. Howie, and F.J. García de Abajo, Phys. Rev. B 60, 11149 (1999).

${ }^{33}$ J.Q. Lu and A.A. Maradudin, Phys. Rev. B 42, 11159 (1990).

${ }^{34}$ L. Spruch, Science 272, 1452 (1996).

${ }^{35}$ R. Fuchs and K.L. Kliewer, J. Opt. Soc. Am. 58, 319 (1968).

${ }^{36}$ R. García-Molina, A. Gras-Martí, A. Howie, and R.H. Ritchie, J. Phys. C 18, 5335 (1985).

${ }^{37}$ F.J. García de Abajo and A. Howie, Phys. Rev. Lett. 80, 5180 (1998).

${ }^{38}$ F.J. García de Abajo, Phys. Rev. B 59, 3095 (1999). 
${ }^{39}$ E. Kröger, Z. Phys. A 235, 403 (1970).

${ }^{40}$ R. Pogorzelski and C. Yeh, Phys. Rev. A 8, 138 (1973).

${ }^{41}$ P. Moreau, N. Brun, C.A. Walsh, C. Colliex, and A. Howie, Phys. Rev. B 56, 6774 (1997).

${ }^{42}$ D.M. Purcell and C.R. Pennypacker, Astrophys. J. 186, 705 (1973).

${ }^{43}$ B.T. Draine and P.J. Flatau, J. Opt. Soc. Am. A 11, 1491 (1994).

${ }^{44}$ R. Rupping, Z. Phys. D: At., Mol. Clusters 36, 69 (1996).

${ }^{45}$ L. Henrard and Ph. Lambin, J. Phys. B 29, 5127 (1996).

${ }^{46}$ A. Rivacoba and F. J. García de Abajo (unpublished).

${ }^{47}$ J.B. Pendry and A. MacKinnon, Phys. Rev. Lett. 69, 2772 (1992).

${ }^{48}$ J.B. Pendry, A.J. Holden, W.J. Stewart, and I. Youngs, Phys. Rev. Lett. 76, 4773 (1996).

${ }^{49}$ J.B. Pendry and L. Martín-Moreno, Phys. Rev. B 50, 5062 (1994).

${ }^{50}$ See for instance D.F. Sievenpiper, E. Yablonovitch, J.N. Winn, S. Fan, P.R. Villeneuve, and J.D. Joannopoulos, Phys. Rev. Lett. 80, 2829 (1998) for a recent example of application.

${ }^{51}$ K.M. Leung and Y.F. Liu, Phys. Rev. Lett. 65, 2646 (1990).

${ }^{52}$ Z. Zhang and S. Satpathy, Phys. Rev. Lett. 65, 2650 (1990).

${ }^{53}$ K. Ohtaka, Phys. Rev. B 19, 5057 (1979).

${ }^{54}$ X. Wang, X.-G. Zhang, Q. Yu, and B.N. Harmon, Phys. Rev. B 47, 4161 (1993).

${ }^{55}$ N. Stefanou, V. Yannopapas, and A. Modinos, Comput. Phys. Commun. 113, 49 (1998); 132, 189 (2000).

${ }^{56}$ W.Y. Zhang, X.Y. Lei, Z.L. Wang, D.G. Zheng, W.Y. Tam, C.T. Chan, and P. Sheng, Phys. Rev. Lett. 84, 2853 (2000).

${ }^{57}$ K. Ohtaka, T. Ueta, and K. Amemiya, Phys. Rev. B 57, 2550 (1998).

${ }^{58}$ L.M. Li and Z.Q. Zhang, Phys. Rev. B 58, 9587 (1998).

${ }^{59}$ F.J. García de Abajo, Phys. Rev. Lett. 82, 2776 (1999); Phys. Rev. B 60, 6086 (1999).

${ }^{60}$ F.J. García de Abajo, Phys. Rev. B 60, 6103 (1999).

${ }^{61}$ N. Ida, Numerical Modeling for Electromagnetic Non-destructive Evaluation (Chapman and Hall, New York, 1995).

${ }^{62} \mathrm{Ph}$. Lambin, A.A. Lucas, and J.-P. Vigneron, Phys. Rev. B 46, 1794 (1992).

${ }^{63}$ Th. Cabioc'h, S. Camelio, L. Henrard, and Ph. Lambin, Eur. Phys. J. B 18, 535 (2000).

${ }^{64}$ M. Kociak, L. Henrard, O. Stéphan, K. Suenaga, and C. Colliex, Phys. Rev. B 61, 13936 (2000).

${ }^{65}$ N. Zabala and P.M. Echenique, Ultramicroscopy 32, 327 (1990).

${ }^{66}$ Z. Zabala, E. Ogando, A. Rivacoba, and F. J. García de Abajo, Phys. Rev. B 64, 205410 (2001).

${ }^{67}$ J.D. Jackson, Classical Electrodynamics (Wiley, New York, 1975).

${ }^{68}$ For an abrupt interface, one has $4 \pi \sigma_{s}=\left(1 / \epsilon_{2}-1 / \epsilon_{1}\right) \mathbf{n}_{s} \cdot \mathbf{D} \delta_{s}$ and $4 \pi \mathbf{m}=\left[i \omega\left(\epsilon_{1} \mu_{1}-\epsilon_{2} \mu_{2}\right) \phi \mathbf{n}_{s}+\left(\mu_{1}-\mu_{2}\right) c \mathbf{H} \times \mathbf{n}_{s}\right] \delta_{s}, \quad$ where the interface normal $\mathbf{n}_{s}$ points towards medium 2 and $\delta_{s}$ is a surface $\delta$ function. The continuity of the scalar potential $\phi$, the normal electric displacement $\mathbf{n}_{s} \cdot \mathbf{D}$, and the tangential magnetic field at the interface provides an unambiguous meaning to these expressions.

${ }^{69}$ There is some freedom in the choice of the boundary charges and currents $\sigma_{j}$ and $\mathbf{h}_{j}$ : the field induced within medium $j$ can be regarded as produced exclusively by the interface charges lying on the side of its boundary $S_{j}$ facing medium $j$, or alternatively, by the whole set of interface charges. The former case is adopted here, so that the boundary charges on $S_{j}$ act as mediators of the interaction between the rest of the boundary charges and medium $j$. Similarly, the volume integrals in Eqs. (6) and (7) can be performed over medium $j$ or they can be safely extended to an infinite space, since the part of these integrals that is outside medium $j$ does not contribute to the left hand side of Eqs. (4) and (5) as a consequence of Eq. (9). This fact can be used to simplify the algebra for penetrating trajectories, in which case the mentioned integrals can be evaluated for infinite homogeneous media containing the entire electron trajectory, rather than by separating the integrals in the parts of the trajectory that connect consecutive interface crossing points.

${ }^{70}$ H. Fearn, D.F.V. James, and P.W. Milonni, Am. J. Phys. 64, 986 (1996).

${ }^{71}$ H. Raether, Surface Plasmons on Smooth and Rough Surfaces and on Gratings (Springer-Verlag, Berlin, 1988), Vol. 111.

${ }^{72}$ S.P. Apell, P.M. Echenique, and R.H. Ritchie, Ultramicroscopy 65, 53 (1996).

${ }^{73}$ D.R. Smith and N. Kroll, Phys. Rev. Lett. 85, 2933 (2000).

${ }^{74}$ C. Wehenkel and B. Gauthé, Solid State Commun. 89, 555 (1974).

${ }^{75}$ E.D. Palik, Handbook of Optical Constants of Solids (Academic Press, New York, 1985).

${ }^{76}$ H. Cohen, T. Maniv, R. Tenne, Y.R. Hacohen, O. Stephan, and C. Colliex, Phys. Rev. Lett. 80, 782 (1998); P.M. Echenique, A. Howie, and R.H. Ritchie, ibid. 83, 658 (1999); H. Cohen et al., ibid. 83, 659 (1999).

${ }^{77}$ M. S. Sander, R. Gronsky, Y. M. Lin, and M. S. Dresselhaus, J. Appl. Phys. 89, 2733 (2001).

${ }^{78}$ J.C. Ashley and L.C. Emerson, Surf. Sci. 41, 615 (1974).

${ }^{79}$ F. Ouyang, P.E. Batson, and M. Isaacson, Phys. Rev. B 46, 15421 (1992).

${ }^{80}$ V. Heine, Solid State Phys. 35, 1 (1980).

${ }^{81}$ R. Haydock, Solid State Phys. 35, 215 (1980).

${ }^{82}$ R. Fuchs (private communication).

${ }^{83}$ M. Hoyles, S. Kuyucak, and S.-H. Chung, Comput. Phys. Commun. 115, 45 (1998). 